\title{
Platelet-RBC interaction mediated by FasL/FasR induces procoagulant activity important for thrombosis
}

\author{
Christoph Klatt, ${ }^{1}$ Irena Krüger, ${ }^{1}$ Saskia Zey, ${ }^{1}$ Kim-Jürgen Krott, ${ }^{1}$ Martina Spelleken, ${ }^{1}$ Nina Sarah Gowert, ${ }^{1}$ Alexander Oberhuber, ${ }^{1}$ \\ Lena Pfaff, ${ }^{2}$ Wiebke Lückstädt, ${ }^{3}$ Kerstin Jurk, ${ }^{4}$ Martin Schaller, ${ }^{5}$ Hadi AI-Hasani, ${ }^{6}$ Jürgen Schrader, ${ }^{7}$ Steffen Massberg, ${ }^{2}$ \\ Konstantin Stark, ${ }^{2}$ Hubert Schelzig, ${ }^{1}$ Malte Kelm, ${ }^{3}$ and Margitta Elvers ${ }^{1}$

\begin{abstract}
'Department of Vascular and Endovascular Surgery, Heinrich-Heine-University University Medical Center, Düsseldorf, Germany. ${ }^{2}$ Medizinische Klinik und Poliklinik I, Klinikum der Universität, Ludwig Maximilians-Universität, Munich, Germany. ${ }^{3}$ Department of Cardiology, Pulmonology and Vascular Medicine, Heinrich-Heine-University, Düsseldorf, Germany and Cardiovascular Research Institute Düsseldorf (CARID), Medical Faculty, University Düsseldorf, Germany. ${ }^{4}$ Center for Thrombosis and Hemostasis (CTH), University Medical Center Mainz, Mainz, Germany. ${ }^{5}$ Department of Dermatology, University of Tübingen, Tübingen, Germany. Institute for Clinical Biochemistry and Pathobiochemistry, German Diabetes Center, Leibniz-Center for Diabetes Research at the Heinrich-Heine-University Düsseldorf, Medical Faculty, Düsseldorf, Germany. Department of Molecular Cardiology, Heinrich-Heine-University, Düsseldorf, Cermany.
\end{abstract}

\begin{abstract}
Red blood cells (RBCs) influence rheology, and release ADP, ATP, and nitric oxide, suggesting a role for RBCs in hemostasis and thrombosis. Here, we provide evidence for a significant contribution of RBCs to thrombus formation. Anemic mice showed enhanced occlusion times upon injury of the carotid artery. A small population of RBCs was located to platelet thrombi and enhanced platelet activation by a direct cell contact via the FasL/FasR (CD95) pathway known to induce apoptosis. Activation of platelets in the presence of RBCs led to platelet FasL exposure that activated FasR on RBCs responsible for externalization of phosphatidylserine (PS) on the RBC membrane. Inhibition or genetic deletion of either FasL or FasR resulted in reduced PS exposure of RBCs and platelets, decreased thrombin generation, and reduced thrombus formation in vitro and protection against arterial thrombosis in vivo. Direct cell contacts between platelets and RBCs via FasL/FasR were shown after ligation of the inferior vena cava (IVC) and in surgical specimens of patients after thrombectomy. In a flow restriction model of the IVC, reduced thrombus formation was observed in $\mathrm{FasL}^{-1-}$ mice. Taken together, our data reveal a significant contribution of RBCs to thrombosis by the FasL/FasR pathway.
\end{abstract}

\section{Introduction}

Platelets play an essential role in hemostasis by the formation of a primary hemostatic plug to avoid excessive blood loss upon vessel injury $(1,2)$. In contrast, uncontrolled platelet activation upon rupture of an atherosclerotic plaque is responsible for acute vessel occlusion, leading to myocardial infarction or stroke $(3,4)$.

Injury of the endothelium leads to exposure of extracellular matrix proteins that serve as substrates to initiate the adhesion and activation of circulating platelets by the engagement of specific receptors (2). vWF binds the platelet-specific GPIb-V-IX complex, collagens bind the glycoprotein receptor GPVI and integrin $\alpha 2 \beta 1$, and fibronectin engages both integrin $\alpha 5 \beta 1$ and laminin $\alpha 6 \beta 1$ (5). vWF binding to GPIb is essential for platelet recruitment to the vessel wall and initial adhesion in arterioles and stenotic arteries (6). Engagement of GPVI by collagen leads to platelet activation important for stable platelet adhesion mediated by integrin $\alpha \operatorname{IIb} \beta 3$ binding to vWF and fibrinogen $(7,8)$ and release of platelet granules. Central for irreversible platelet activation

Related Commentary: p. 3747

Conflict of interest: The authors have declared that no conflict of interest exists. Submitted: December 1, 2016; Accepted: June 21, 2018

Reference information: J Clin Invest. 2018;128(9):3906-3925

https://doi.org/10.1172/JCI92077. is the release of soluble agonists such as thromboxane and ADP because they increase platelet activation by binding to $\mathrm{G}$ proteincoupled receptors $(9,10)$.

Primary hemostasis is essential for a first coverage of injured vessels but not sufficient for thrombus growth and stability (5). Platelet activation followed by blood coagulation and thrombin generation is crucial for the formation of stable arterial thrombi. Platelets play an important role in these processes because they provide a procoagulant surface by exposing phosphatidylserine (PS) to allow assembly of coagulation complexes on their plasma membrane. So far, it is known that PS is exposed by the protein scramblase, which does only require an increased $\mathrm{Ca}^{2+}$ concentration but no energy, and simultaneous $\mathrm{Ca}^{2+}$-induced inactivation of translocase and flippase. This happens, for example, during early apoptosis (11). Platelets support fibrin formation and regulate the retraction of fibrin clots (12).

Red blood cells (RBCs) have been described as participating in hemostasis by influencing rheology. In flowing blood, platelets are pressed against the vessel wall leading to a higher concentration of platelets. Thus, an increase in the hematocrit is accompanied by improved hemostasis, especially upon anemia (13-16). In 1961, Hellem and colleagues cited platelet adhesiveness as the most important platelet function in hemostasis (13). The authors cite a study from Duke from 1910 in which direct transfusions were administered to patients with anemia 
A

Whole blood

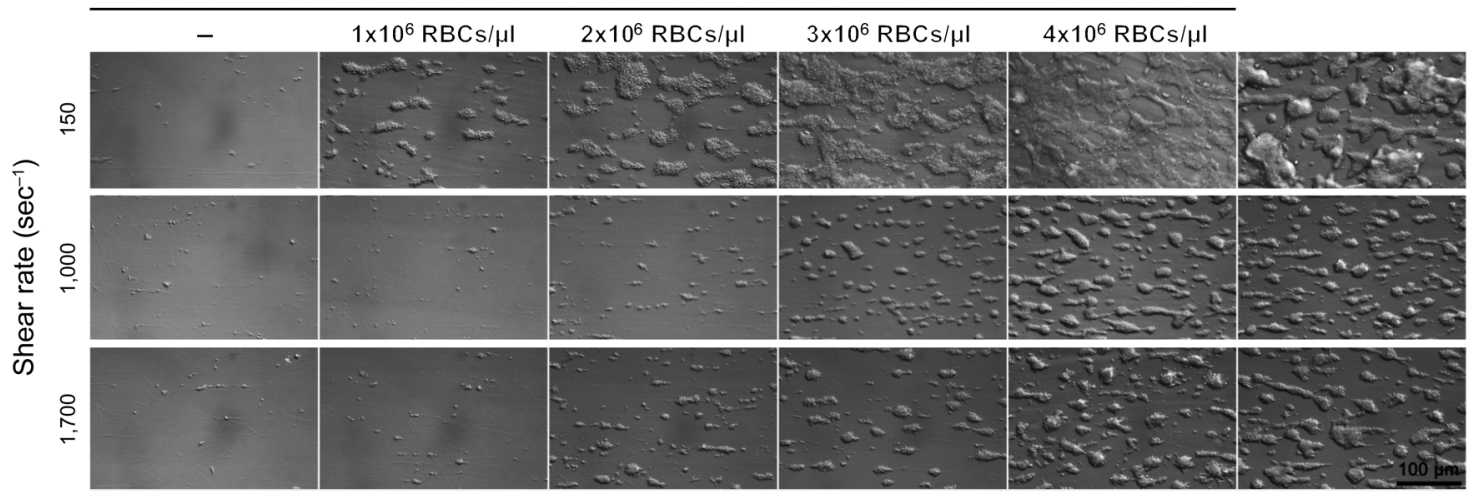

B

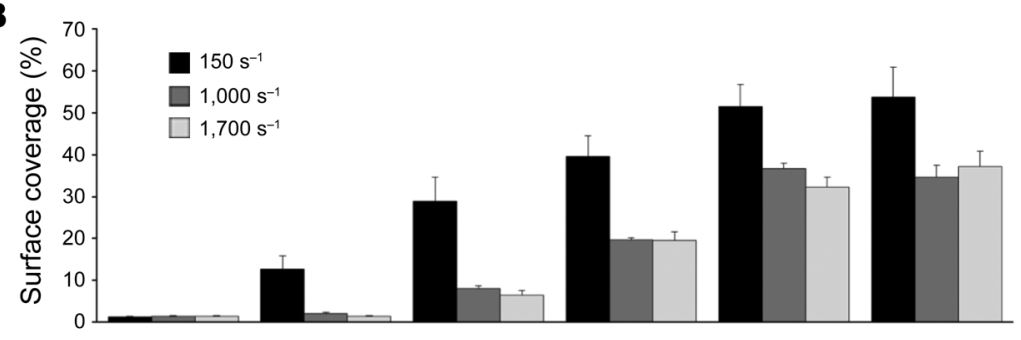

C

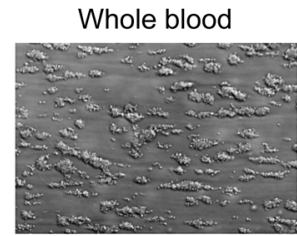

$\mathrm{PRP}+7 \times 10^{3} \mathrm{WBCs}$

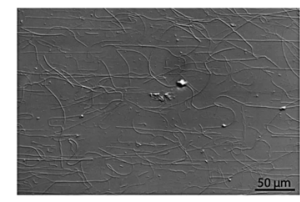

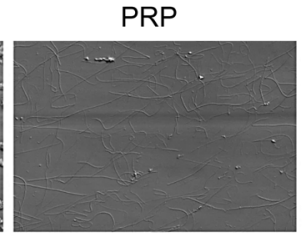

$\mathrm{PRP}+>15 \times 10^{4} \mathrm{WBCs}$

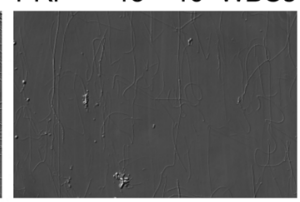

D

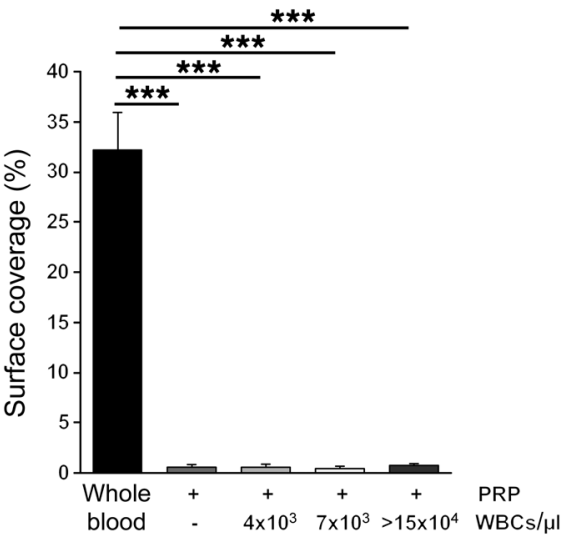

E

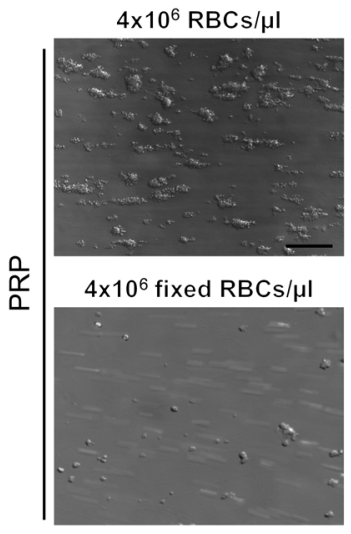

$\mathbf{F}$

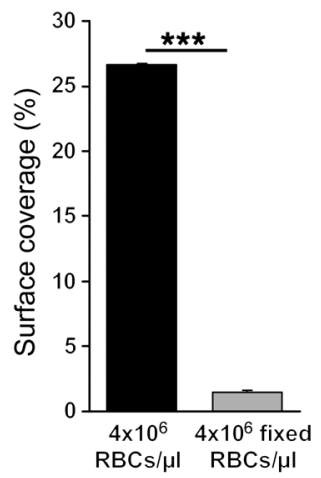

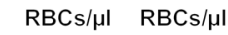

Figure 1. RBCs are essential for 3-dimensional thrombus formation under flow ex vivo. Human whole blood or PRP supplemented with different concentrations of RBCs was perfused over a collagen-coated surface at indicated shear rates. The effects of blood components on thrombus formation, as indicated by mean surface coverage, were determined. Data are expressed as arithmetic means. (A) Representative phase-contrast images at the end of the perfusion period. Scale bar: $100 \mu \mathrm{m}$. (B) Quantification of surface coverage with increasing RBC concentrations (from $1 \times 10^{6}$ to $4 \times 10^{6} \mathrm{RBCs} / \mu \mathrm{l}$, left to right) added to PRP in comparison with whole blood. Data are given for 3 shear rates of $150 \mathrm{~s}^{-1}$ (black column), 1,000 s-1 (gray column), and 1,700 s-1 (white column). Blood components as indicated below and shear rates as shown on the left. (C) Effect of WBCs on thrombus formation under flow. Representative images of surface coverage of whole blood and PRP in the absence and presence of WBCs $\left(7 \times 10^{3} \mathrm{WBCs} / \mu \mathrm{l}\right.$ and $>15 \times 10^{4} \mathrm{WBCs} / \mu \mathrm{l}$, respectively). Scale bar: $50 \mu \mathrm{m}$. (D) Quantitative analysis of surface coverage per visual field. (E and F) Thrombus formation using PRP and fixed RBCs. Bar graphs depict mean values \pm SEM. $n=3$, all experiments. ${ }^{* *} P<0.001$ by Student's $t$ test. PRP, platelet-rich plasma; RBC, red blood cell.

and thrombocytopenia. They claimed that RBCs influence the hemostatic mechanism because bleeding time in patients with severe anemia was prolonged. Livio and colleagues investigated uremic patients who are often anemic (15). They observed that bleeding times in uremic patients are profoundly influenced by anemia. Blajchman et al. were able to confirm the clinical data in experimental animals (17). They found that RBC transfusions shorten bleeding time in thrombocytopenic rabbits. Saniabadi proposed in 1985 that manipulating the hematocrit is a useful therapeutic option in the control of thrombotic and bleeding tendencies (18). Today, the German guidelines for polytrauma and treatment of seriously injured persons (Kurzversion der S3 - Leitlinie Polytrauma/Schwerverletzten-Behandlung) dictate that the hematocrit be increased to a minimum 7-9 g/dl (Chap- 
A

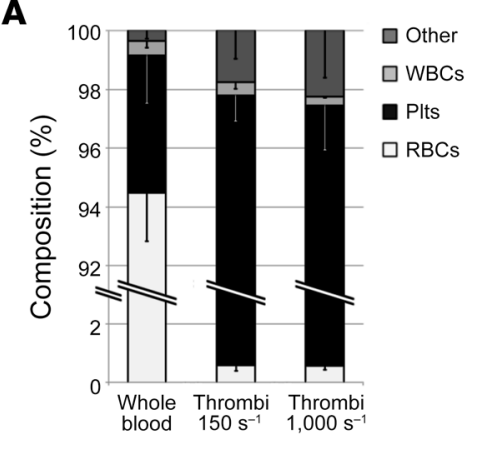

C

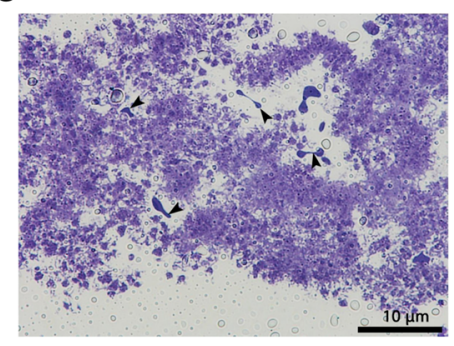

D

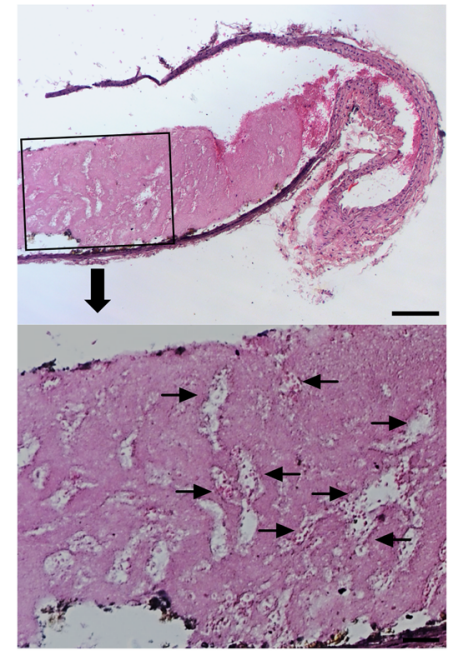

H

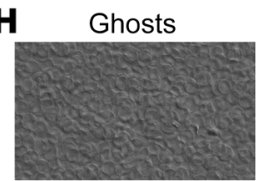

Ghosts 1:10 diluted

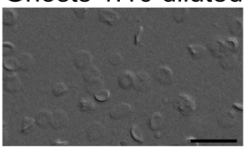

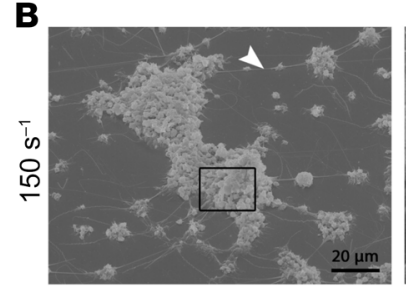
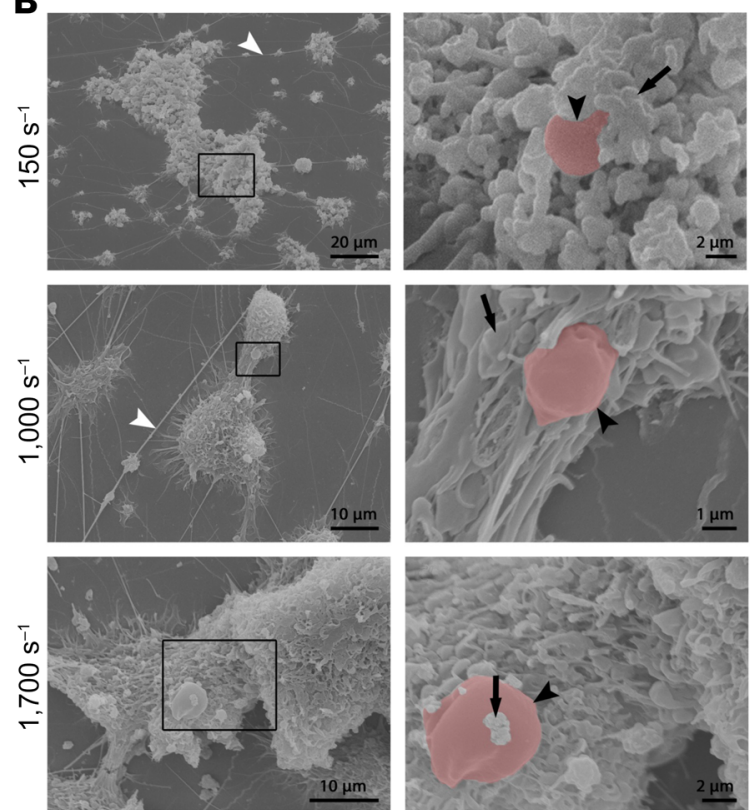

E

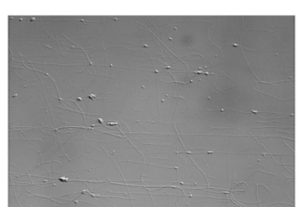

$4 \times 10^{6} \mathrm{RBCs}$

Hemoglobin
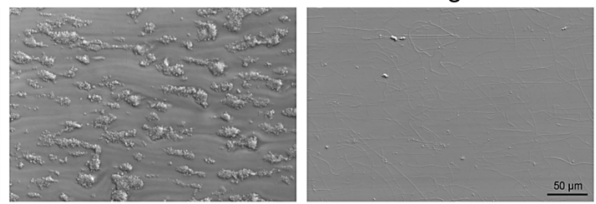

$\mathbf{F}$

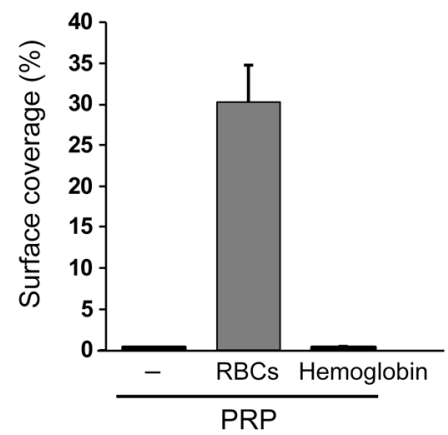

G
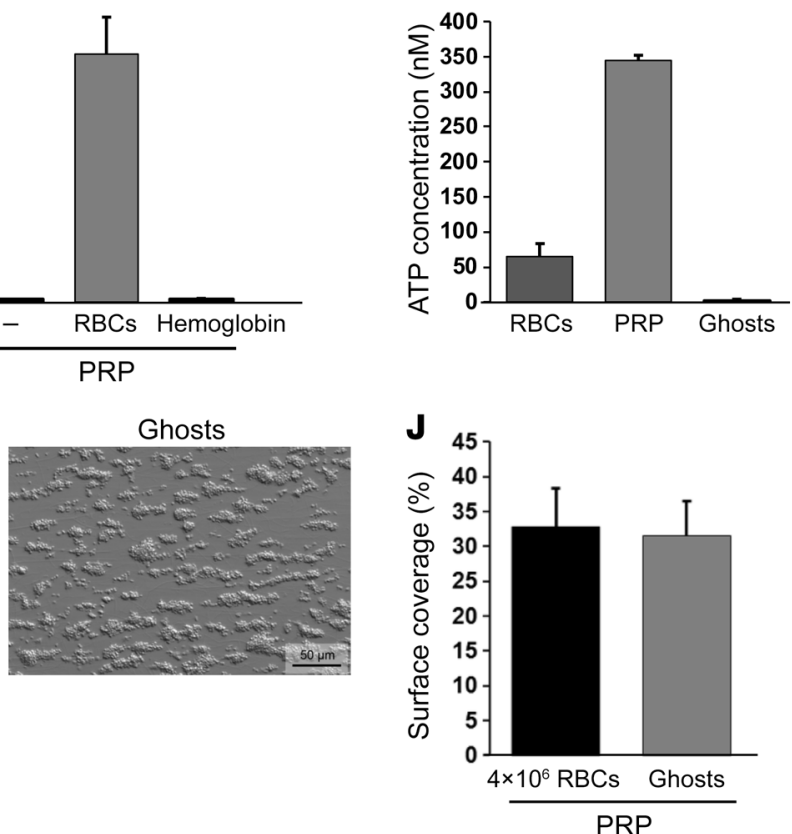

PRP
I $4 \times 10^{6} \mathrm{RBCs}$

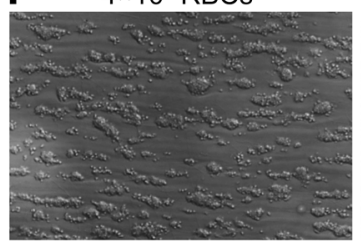


Figure 2. A small population of RBCs in platelet-rich thrombi is sufficient to support thrombus formation. (A-D) Cellular composition of whole blood and thrombi. (A) Cell types were determined by morphology and cell-specific antibodies in flow cytometry. The diagram presents the percentage of RBCs (white), platelets (black), WBCs (light gray), and cells that could not be precisely identified (other, dark gray) $(n=3)$. (B) Semithin section through a middle layer of a thrombus. RBCs, some indicated by black arrowheads, are spread throughout the thrombus. Scale bar: $10 \mu \mathrm{m}$. (C) Scanning electron microscopy of thrombi. Collagen fibers indicated by white arrowheads, platelets indicated by black arrows, and RBCs indicated by black arrowheads $(n=3)$. Scale bars as indicated.

(D) Thrombi from mice after injury of the carotid artery were isolated and stained with hematoxylin and eosin to visualize RBCs in arterial thrombi. Representative images are shown. Overview in upper panel, details in lower panel. Scale bars: $100 \mu \mathrm{m}$ (upper panel) and $20 \mu \mathrm{m}$ (lower panel). (E and $\mathbf{F}$ ) Effects of hemoglobin on thrombus formation ex vivo. (E) Representative images. Scale bar: $50 \mu \mathrm{m}$. (F) Mean surface coverage for PRP (2 $\times 10^{5}$ platelets $\left./ \mu \mathrm{l}\right), \mathrm{PRP}+\mathrm{RBCs}\left(4 \times 10^{6} \mathrm{RBC} / \mu \mathrm{l}\right)$, and PRP + hemoglobin $(13 \mathrm{mg} / \mathrm{ml})$ at a shear rate of $1,000 \mathrm{~s}^{-1}(n=3)$. (C) ATP release from RBCs, platelets, and ghosts was induced by L-arginine and detected by HPLC. A total of $4 \times 10^{9} \mathrm{RBC}$, an equivalent amount of ghosts, and $2 \times 10^{8}$ platelets were used $(n=3)$. (H) Ghost preparation was visualized by microscopy for quality inspection. Scale bar: $50 \mu \mathrm{m}$. (I and J) Thrombus formation in the presence of RBCs or ghosts and PRP at a shear rate of 1,000 s-1. (I) Representative images. Scale bar: $50 \mu \mathrm{m}$. (J) Mean surface coverage per visual field $(n=3)$. Bar graphs depict mean values \pm SEM. Plts, platelets; WBCs, white blood cells. Student's $t$ test (J).

ter 2.16, 2.108) and that massive transfusion therapy be induced with fresh frozen plasma, RBCs, and platelets at the ratio of 4:4:1 (Chapter 2.16, 2.110).

Besides rheological effects, different mechanisms underlying RBCs' ability to influence hemostasis have been described, e.g., by the supply of ADP, ATP, or NO (19-22). Recent data provide evidence that RBCs are able to expose PS on their membrane and contribute to the generation of thrombin (23-25). However, a direct contribution of RBCs to arterial thrombus formation and the impact of RBCs in platelet activation and thrombus formation are not well defined. The present study thus explored the active role of RBCs and the relevant mechanisms critical for platelet function and thrombus formation in vitro and in vivo.

\section{Results}

$R B C s$ are essential for 3-dimensional thrombus formation under flow ex vivo. To investigate RBC-driven mechanisms in thrombus formation, we analyzed platelet adhesion and thrombus formation under flow using a flow chamber system. Platelet-rich plasma (PRP) was supplemented with different concentrations of RBCs $\left(1 \times 10^{6}\right.$ to $4 \times 10^{6} \mathrm{RBCs} / \mu \mathrm{l} ; 4 \times 10^{6} \mathrm{RBCs} / \mu \mathrm{l}$ corresponds to a physiological hematocrit of $40 \%$; Figure $1, \mathrm{~A}$ and B, Supplemental Figure 1, and Supplemental Videos 1 and 2; supplemental material available online with this article; https://doi.org/10.1172/ JCI92077DS1) or white blood cells (WBCs, Figure 1, C and D) and perfused over a collagen-coated surface at different wall shear rates $\left(150,1,000\right.$, and $\left.1,700 \mathrm{~s}^{-1}\right)$. No thrombus formation was observed with PRP alone or PRP supplemented with different concentrations of WBCs $\left(4 \times 10^{3}, 7 \times 10^{3}, 75 \times 10^{3}\right.$, and $>15 \times 10^{4}$ WBCs/ $\mu \mathrm{l}$; shear rate $1,000 \mathrm{~s}^{-1}$; Figure $1, \mathrm{C}$ and D, and Supplemental Figure 1). Moreover, no alterations in thrombus formation were detected with $2 \times 10^{6}$ and $4 \times 10^{6} \mathrm{RBCs}$ supplemented with $75 \times 10^{3}$ WBCs compared with RBCs alone (shear rate $1,000 \mathrm{~s}^{-1}$; Supplemental Figure 1). The formation of aggregates and 3-dimensional thrombi increased with increasing amounts of RBCs. Interestingly, the impact of RBCs in thrombus formation was shown to be shear-rate dependent because the addition of $1 \times 10^{6} \mathrm{RBCs} / \mu \mathrm{l}$ to PRP led to the formation of thrombi and PRP supplemented with $2 \times 10^{6} \mathrm{RBCs} / \mu \mathrm{l}$ resulted in robust thrombus growth under a shear rate of $150 \mathrm{~s}^{-1}$, while no thrombus formation $\left(1 \times 10^{6} \mathrm{RBCs} / \mu \mathrm{l}\right)$ or only aggregate formation $\left(2 \times 10^{6} \mathrm{RBCs} / \mu \mathrm{l}\right)$ was observed under a shear rate of 1,000 and $1,700 \mathrm{~s}^{-1}$, respectively. The addition of $4 \times 10^{6} \mathrm{RBCs} / \mu \mathrm{l}$ to PRP induced 3-dimensional thrombus formation, as observed with whole blood that served as positive control (Supplemental Videos 1 and 3). In contrast, high platelet concentrations (PRP with $4 \times 10^{6}$ platelets/ $\mu$ l) did not result in robust thrombus formation under flow (Supplemental Figure 1). Because RBCs play an important role in rheology, we performed fixation of $4 \times 10^{6} / \mu \mathrm{l} \mathrm{RBCs}$ and perfused them together with PRP through the chamber to preserve rheology but to avoid any responses of RBCs upon blood perfusion. Again, we did not observe 3-dimensional thrombus formation and only platelet aggregates were detected (Figure 1, E and F). These data suggest that RBCs might have an active role in thrombus formation. Raising the hematocrit to $75 \%$ $\left(8 \times 10^{6} \mathrm{RBCs} / \mu \mathrm{l}\right)$ did not further increase platelet adhesion and thrombus formation (Supplemental Figure 1, D and E) and the addition of old RBCs ( $>40$ days after blood withdrawal) did not result in altered thrombus formation under flow (Supplemental Figure 1, $\mathrm{F}$ and $\mathrm{G}$ ).

A small population of RBCs in platelet-rich thrombi is sufficient to support thrombus formation under flow. To characterize the impact of RBCs in thrombus formation under flow, we compared the cellular components of whole blood and thrombi that were formed under flow conditions. Blood cells in thrombi were dissolved in Accutase to isolate individual cells of the thrombus and analyzed by flow cytometry according to cell morphology and cell-specific membrane proteins using cell-type-specific antibodies (Figure 2A). While greater than $94 \%$ of cells in whole blood are of RBC origin, we only found less than $2 \%$ RBCs in thrombi that were formed under flow conditions using whole blood and shear rates of 150 and $1,000 \mathrm{~s}^{-1}$. The analysis of thrombi by toluidine blue staining (Figure 2B) and scanning electron microscopy (SEM; Figure 2C) revealed that only very few RBCs are incorporated into a thrombus, confirming the data from flow cytometry. Analysis of thrombi isolated from C57BL/6 mice that underwent $\mathrm{FeCl}_{3}$-induced injury of the carotid artery showed that a small number of RBCs are located within a thrombus also in vivo (Figure 2D). In fact, these RBCs are located in small islands within the thrombus (Figure 2D).

To test if hemoglobin influences thrombus formation under flow, we perfused PRP supplemented with $13 \mathrm{mg} / \mathrm{ml}$ hemoglobin through the chamber and analyzed thrombus formation (Figure 2, E and F). No thrombus formation was observed. However, control experiments revealed that no hemoglobin was released upon platelet adhesion and thrombus formation under flow (Supplemental Figure 1).

To investigate the molecular mechanisms of RBCs and platelets important for thrombus formation, we prepared ghosts. Ghosts have an intact membrane but do not contain any metab- 
A

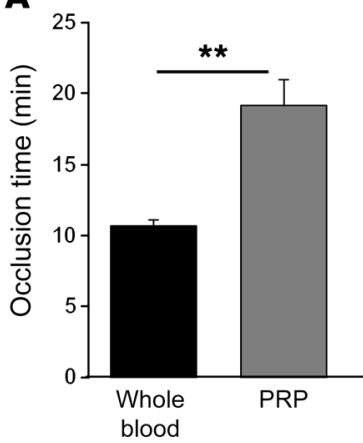

B

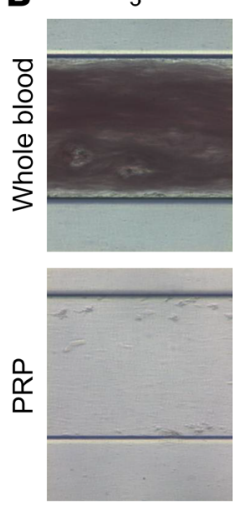

7
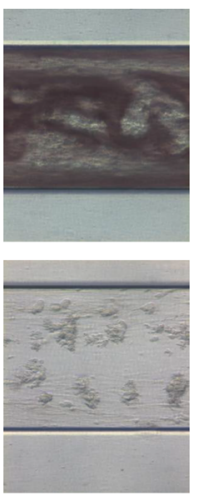

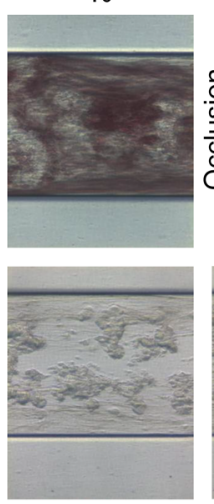

15
19 Time (min)
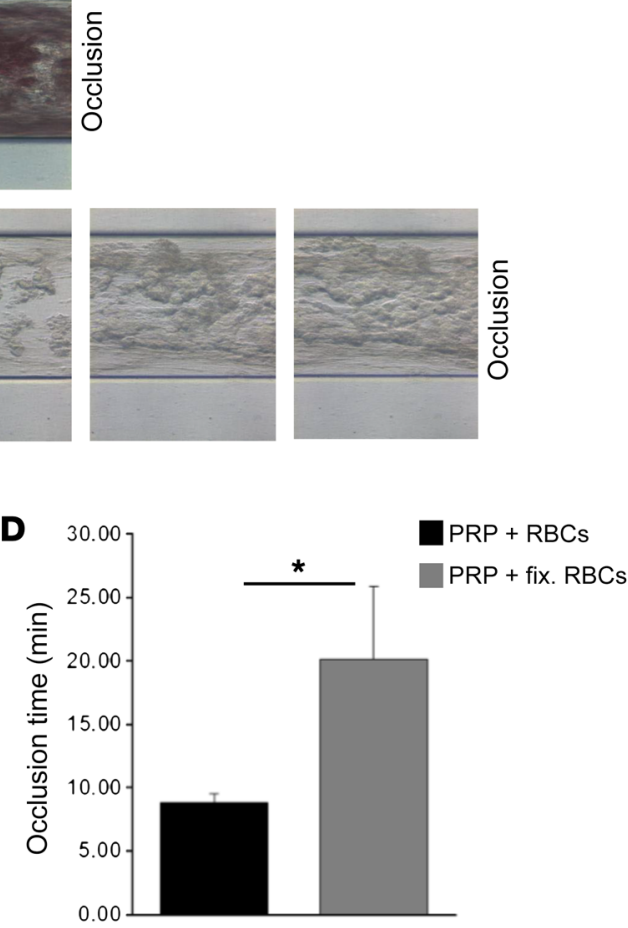

G

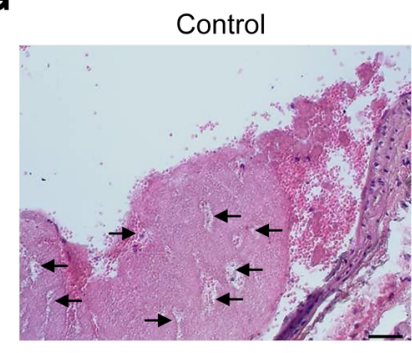

Anemic

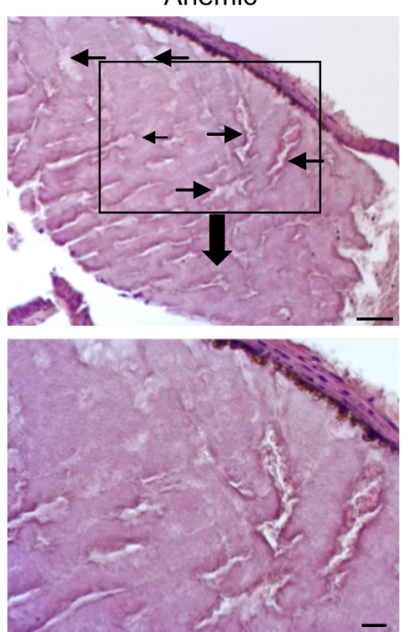


Figure 3. RBCs support occlusive thrombus formation ex vivo and in vivo. (A) Flow chamber system T-TAS. Whole blood and PRP were loaded into the flow chamber at a shear rate of $600 \mathrm{~s}^{-1}$ at $37^{\circ} \mathrm{C}$. Bar graphs show full occlusion of the capillary $(n=6)$. (B) Representative images of whole blood and PRP are shown. Blood flow direction was from left to right. (C) Whole blood and PRP were measured until full occlusion of the capillary. When flow pressure increased to $80 \mathrm{kPa}$, blood perfusion stopped and the capillary was almost completely occluded by thrombi. (D) Human PRP and RBCs (4 $\times$ $\left.10^{6}\right)$ were perfused through the flow chamber. Where indicated, RBCs were substituted with fixed RBCs and occlusion of the capillary was monitored. (E) Bleeding times of control and anemic mice after amputating the tail tip. The time from the incision to the cessation of bleeding was recorded (no blood flow for 1 minute). (F) Time to initiation of thrombus formation (left) and time to occlusion (right) were measured with an ultrasonic flow probe in mice where the carotid artery was injured by topical application of $\mathrm{FeCl}_{3}$ $(n=5-6)$. (C) After injury of the carotid artery, thrombi were isolated and stained with hematoxylin and eosin to visualize RBCs within the thrombus. Representative images of control mice (upper panel, same control sample but different detail as shown in Figure 2D) and anemic mice (middle and lower panel) are shown. Scale bars: $50 \mu \mathrm{m}$ (upper and middle panel) and 20 $\mu \mathrm{m}$ (lower panel). (H) Bleeding times of mice that received RBC injections to increase the hematocrit were analyzed. (I) Time to occlusion in mice with increased hematocrit was determined after injury of the carotid artery with $\mathrm{FeCl}_{3}(n=9-10) . \mathrm{HCT}+$, mice with increased hematocrit. Data are the mean \pm SEM. ${ }^{*} P<0.05,{ }^{* *} P<0.01$ by Student's $t$ test.

olites such as NO or ADP/ATP. Measurements of ATP levels in ghosts confirmed successful preparation (Figure 2, G and $\mathrm{H}$ ). Flow chamber experiments using PRP and ghosts showed platelet adhesion and thrombus formation comparable to experiments where PRP and fresh RBCs $\left(4 \times 10^{6} \mathrm{RBCs} / \mu \mathrm{l}\right)$ were used (Figure 2, I and J, and Supplemental Videos 3 and 4), suggesting that components of the plasma membrane of RBCs and not metabolites of RBC origin play an important role in platelet thrombus formation. This was further supported by unaltered ATP levels in native plasma compared with the flow through in the perfusion system (Supplemental Figure 2).

$R B C$ support occlusive thrombus formation ex vivo and in vivo. In a further set of experiments, thrombus formation was investigated in another ex vivo model, where occlusion of capillaries was analyzed (Figure 3, A-D). In all experiments where PRP was used, full occlusion of the collagen-coated capillaries was significantly delayed compared with whole blood. Full occlusion of the capillaries was achieved within $10.68 \pm 0.42$ minutes using whole blood compared with $19.16 \pm 1.82$ minutes using PRP $(P$ $=0.0027)$. Because PRP and whole blood have different total cell concentrations we also performed experiments with PRP and fixed RBCs $\left(4 \times 10^{6}\right)$ to avoid differences in rheology (Figure 3D, unaltered size of fixed RBCs is shown in Supplemental Figure 3A). Again, we measured significantly delayed occlusion of the capillary where PRP and fixed RBCs were used compared with PRP and fresh untreated RBCs $\left(4 \times 10^{6} ; 8.86 \pm 0.64\right.$ minutes using PRP and fresh RBCs compared with $20.14 \pm 5.71$ minutes using PRP and fixed RBCs, $P=0.0486$ ).

To investigate the impact of RBCs in hemostasis and arterial thrombus formation in vivo, we used anemic mice. To achieve anemia, $200 \mu \mathrm{l}$ blood was taken from C57BL/6 mice on 2 consecutive days to reduce the hematocrit to $21 \%-28 \%$ (ca. $10 \mathrm{~g} / \mathrm{dl}$, Supplemental Figure 3, B and C). We then transfused PRP alone or PRP (up to $45 \times 10^{4} / \mu \mathrm{l}$ ) with RBCs (number of RBCs until a hematocrit of $\sim 40 \%$ was reached) to anemic mice (total platelet counts in the circulation of control and anemic mice were similar after transfusion) and analyzed tail bleeding times as well as arterial thrombus formation in vivo. The determination of bleeding times by amputating the tail tip of mice resulted in arrest of bleeding after 144.2 \pm 11.2 (PRP + RBCs) versus $515.3 \pm 112.6$ (PRP) seconds, providing strong evidence that increased hematocrit is important for normal hemostasis (Figure 3E). To investigate if reduced hematocrit affects occlusive arterial thrombus formation, the right carotid artery was injured by topical application of $15 \% \mathrm{FeCl}_{3}$ and blood flow was monitored by an ultrasonic flow probe. As shown in Figure 3F, the onset of thrombus formation as detected by the formation of small aggregates was not different between control and anemic mice, although a slight but not significant increase was measured in anemic mice (mean time: PRP + RBCs $245 \pm 61.8$ seconds; PRP $420 \pm 74.5$ seconds, $P=0.1207$ ). However, anemic mice showed significantly enhanced occlusion times compared with control mice (mean time: $\mathrm{PRP}+\mathrm{RBCs} 537.5$ \pm 111.3 seconds; PRP $1,005 \pm 56.1$ seconds, $P=0.009$, Figure $3 \mathrm{~F}$ ), suggesting that the hematocrit influences platelet adhesion and aggregate formation at the injured vessel wall in vivo. Accordingly, only very few RBCs were present within the thrombus of anemic mice compared with control mice (Figure 3G). To investigate if increased hematocrit affects hemostasis or arterial thrombosis, we increased the hematocrit ( $>50 \%$; Supplemental Figure 3D) in mice by intravenous injection of fresh RBCs from donor mice from the same genotype and determined bleeding times in these mice (Figure $3 \mathrm{H})$. Mice with increased hematocrit (HCT+) did not show altered hemostasis compared with control mice. Along these lines, arterial thrombosis was not altered in mice with increased hematocrit, as shown by comparable occlusion times in mice with increased hematocrit and control mice after $\mathrm{FeCl}_{3}$-induced injury of the carotid artery (Figure 3I).

$R B C s$ facilitate thrombin generation and PS exposure of RBCs is important for arterial thrombus formation on collagen. RBCs are known to expose PS on their membrane and contribute to the generation of thrombin (23-25). To elucidate whether RBCs provide a procoagulant surface we performed flow chamber experiments with PRP and $4 \times 10^{6} / \mu \mathrm{l}$ RBCs or ghosts and incubated ghosts with annexin $\mathrm{V}$ before perfusion to neutralize exposed PS on the surface of cells (26). As a result, thrombus formation was strongly reduced when annexin $\mathrm{V}$ was added to ghosts before PRP and ghosts were mixed and perfused through the flow chamber (Figure 4, A and B). To examine the impact of PS of intact cells, we incubated RBCs with ionomycin to induce PS exposure. Thrombus formation with PRP and ionomycin-treated RBCs was not different from PRP supplemented with fresh RBCs. In contrast, ionomycin-treated RBCs showed significantly reduced thrombus formation under flow when pretreated with annexin $\mathrm{V}$ (Figure $4 \mathrm{C}$ ). In a flow cytometric analysis we confirmed binding of annexin $\mathrm{V}$ to ghosts, RBCs treated with ionomycin, and old RBCs ( $>40$ days after blood withdrawal), but not to fresh RBCs (Figure 4D). A detailed characterization of the different RBC/CD235-positive cells used in this study is provided in Supplemental Figure 4. Moreover, we measured PS exposure of RBCs and ghosts after isolation of cells by Accutase treatment of thrombi at the end of the flow chamber experiment. As expected, increasing the concentra- 
A

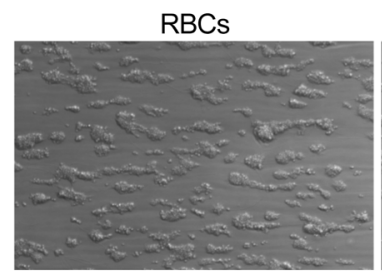

B

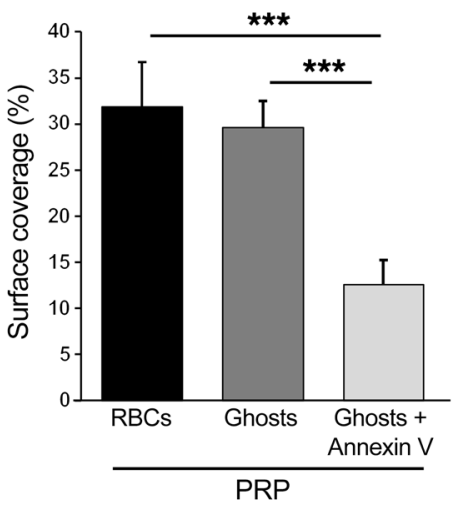

D
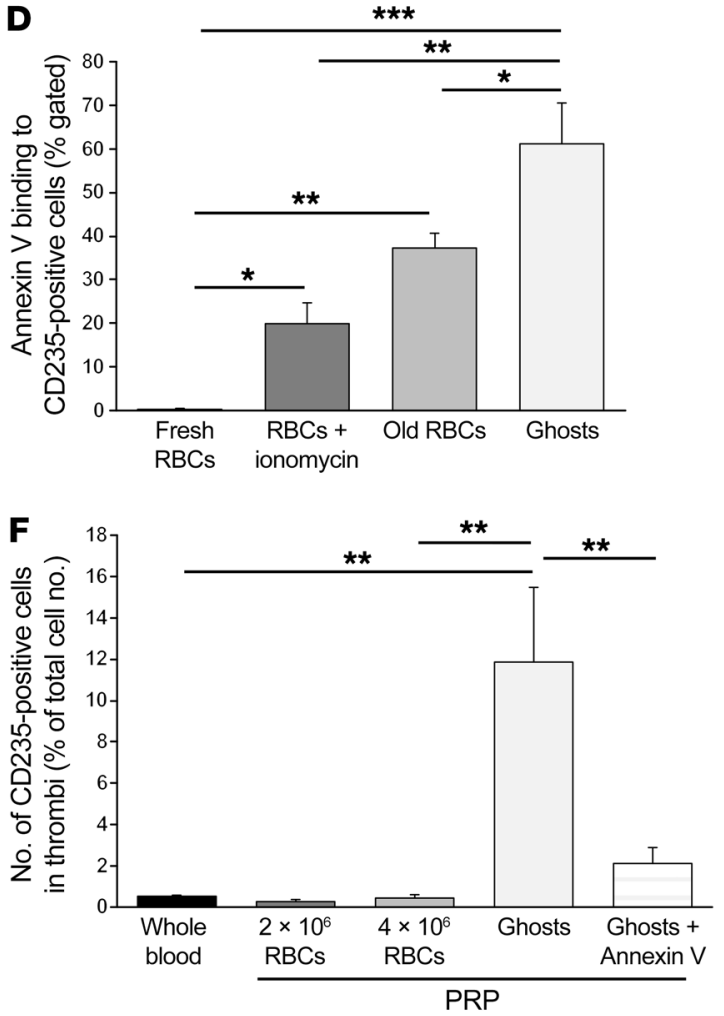

Ghosts

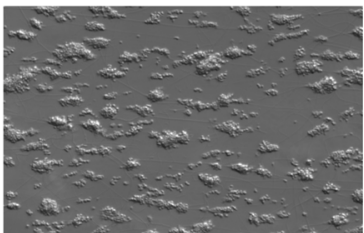

C

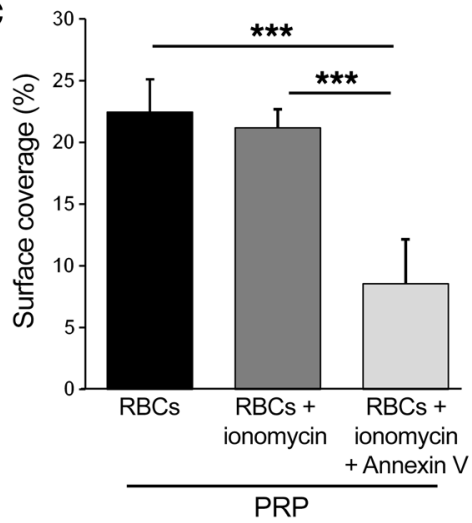

Ghosts + Annexin V

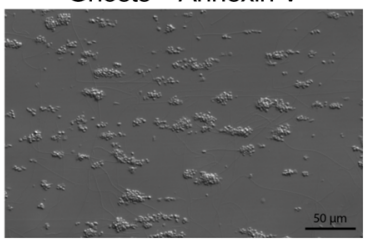

E

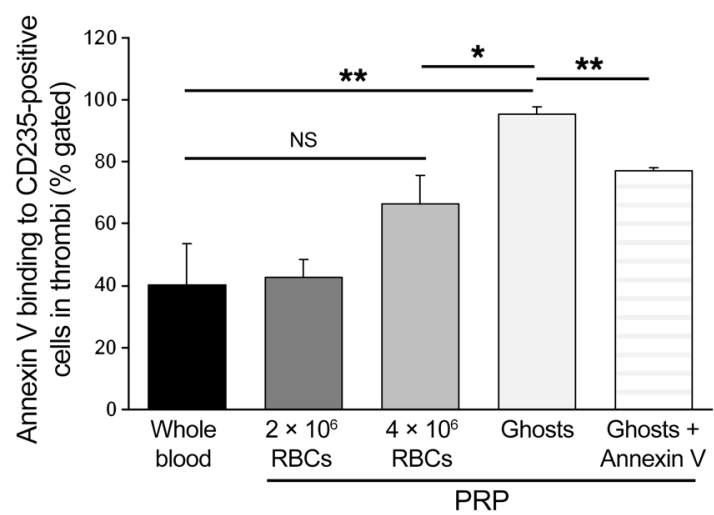

G

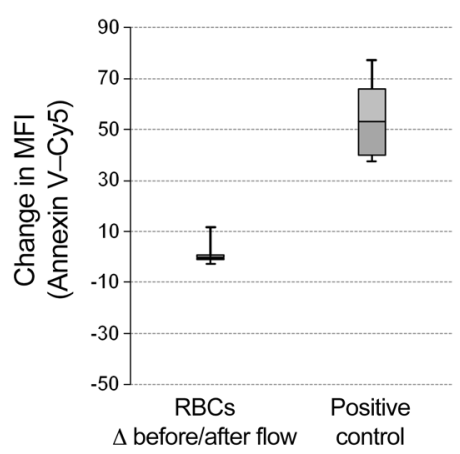

H

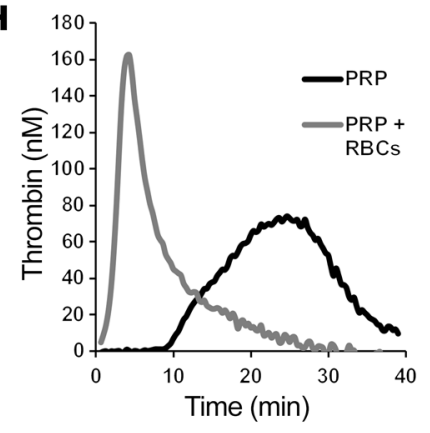

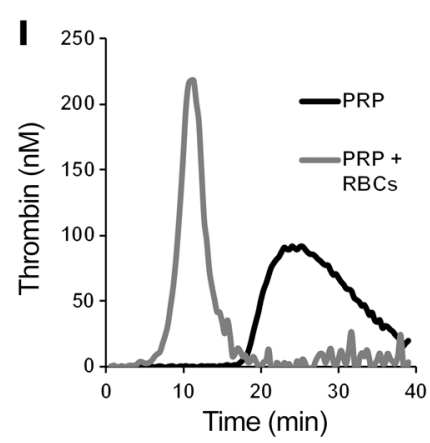

J

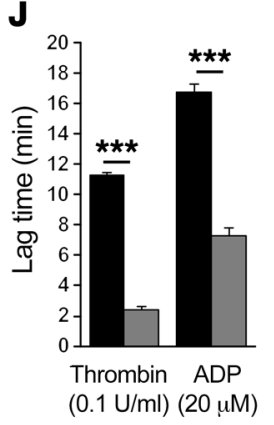

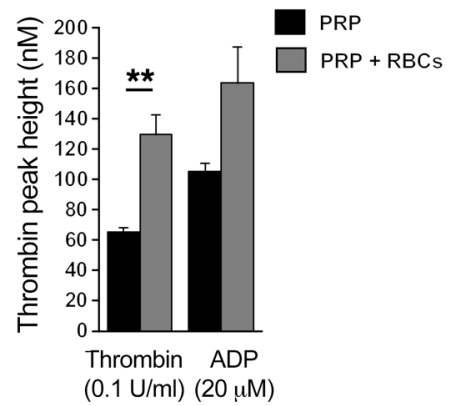


Figure 4. PS exposure of RBCs is responsible for RBC-mediated 3-dimensional thrombus formation on collagen. ( $A$ and $\mathbf{B}$ ) Thrombus formation on collagen under flow using PRP supplemented with freshly isolated RBCs, ghosts, and ghosts incubated with annexin $V$ to neutralize $P S$ at a shear rate of $1,000 \mathrm{~s}^{-1}$. Representative images (A) with blood components as indicated above. (B) Thrombus formation was quantified by surface coverage per visual field $(n=3)$. Scale bar: $50 \mu \mathrm{m}$. (C) PS exposure of RBCs was induced by treatment with ionomycin. Defective aggregate formation under flow using PRP and RBCs treated with ionomycin and annexin V. Mean surface coverage per visual field (left panel) $(n=3)$. Representative image of PRP supplemented with RBCs treated with ionomycin and annexin $V$ (right panel). Scale bar: $50 \mu \mathrm{m}$. (D) Annexin $V$ binding to fresh RBCs, RBCs treated with ionomycin, RBCs after storage for more than 40 days (old RBCs), and ghosts was measured by flow cytometry $(n=3)$. (E) Determination of annexin $\mathrm{V}$ binding to RBCs and ghosts isolated from thrombi developed in the flow chamber system $(n=3)$. Where indicated, ghosts were preincubated with annexin V. (F) Number of CD235-positive cells in thrombi formed under flow was determined via flow cytometry. (C) Annexin $\mathrm{V}$ binding to RBCs before and after the flow through as determined by change in mean fluorescence intensity (MFI) compared with PS exposure of ghosts (positive control). (H-J) Thrombin- (H) and ADP-

induced (I) thrombin generation in PRP and PRP supplemented with RBCs was measured with the fluorogenic calibrated automated thrombogram assay. (J) Lag time (minutes, left panel) and peak height (nM thrombin, right panel) are shown. Data are expressed as arithmetic means \pm SEM. Student's $t$ test ( $\mathbf{A}$ and $\mathbf{J}$ ) and 1-way ANOVA with Tukey's multiple-comparisons test (B-F). ${ }^{*} P<0.05$; ${ }^{* *} P<0.01$; ${ }^{* *} P<0.001$. Cy5, cyanine 5; NS, not significant; PS, phosphatidylserine.

tion of RBCs in the flow chamber led to a stronger annexin $\mathrm{V}$ signal in flow cytometry. The analysis of ghosts revealed high PS exposure of these cells that was significantly reduced by pretreatment of ghosts with annexin $\mathrm{V}$ before starting the flow chamber run (Figure 4E). In control experiments, we confirmed that treatment with Accutase leads to almost complete dissolution of cells from the coverslip and we were able to exclude any effect of Accutase treatment on PS exposure of RBCs (Supplemental Figure 5). Flow cytometric analysis confirmed an increased number of CD235positive cells when we perfused ghosts through the flow chamber compared with different approaches with fresh RBCs or pretreatment of ghosts with annexin $\mathrm{V}$ (Figure 4F). The change in mean fluorescence intensity (MFI) of annexin V binding to RBCs demonstrated almost no differences when comparing RBCs before perfusion through the chamber and thereafter (RBCs that leave the chamber without being recruited to the thrombus, Figure 4G). These data confirmed that most RBCs within a thrombus expose PS on their surface, while the vast majority of RBCs from the flow through do not expose PS (Figure 4, E-G).

Next, we determined thrombin generation in PRP and PRP supplemented with RBCs after stimulation of platelets with either thrombin (Figure 4, $\mathrm{H}$ and J) or ADP (Figure 4, I and J). Thrombin was generated faster, as demonstrated by shorter lag time, and the thrombin peak was increased when PRP was supplemented with RBCs compared with PRP alone (Figure $4, \mathrm{H}-\mathrm{J}$ ).

Cell-cell contact between platelets and RBCs is crucial for RBCplatelet interaction. To determine which signal or mechanism is responsible for the induction of PS externalization of RBCs, we perfused whole blood through the flow chamber and allowed thrombus formation on a collagen matrix. Thrombi were then analyzed by transmission electron microscopy (TEM). As shown in Figure $5 \mathrm{~A}, \mathrm{RBCs}$ are located within the thrombus, as already shown in Figure 2. Furthermore, we found RBCs close to platelets and with direct contact to platelets. Flow cytometric analysis of static experiments using PRP incubated with fresh RBCs demonstrated that a small subpopulation of RBCs and platelets interact with each other because these cells were positively labeled for the platelet marker GPIb $\alpha$ and the RBC marker CD235a (Figure 5B). Comparable results were obtained with ADP-stimulated platelets (data not shown). To disclose the underlying mechanisms, we incubated RBCs with the supernatant of collagen-related peptidestimulated (CRP-stimulated) platelets and measured annexin V binding of RBCs. As expected, no increase in annexin $\mathrm{V}$ binding was observed (Figure 5C). However, experiments using PRP and thus intact platelets revealed a significant increase in PS exposure of RBCs, providing further evidence that a direct cell contact between platelets and RBCs is necessary to induce PS exposure of RBCs. Further experiments using aspirin and clopidogrel to inhibit thromboxane formation or ADP signaling via the ADP receptor $\mathrm{P}_{2} \mathrm{Y}_{12}$, respectively, demonstrated that neither thromboxane nor $\mathrm{P} 2 \mathrm{Y}_{12}$ plays a major role in platelet-mediated induction of PS exposure of RBCs (Supplemental Figure 6A). All these data prompted us to look for a receptor-mediated interaction between platelets and RBCs. Recently, the platelet receptors CD36 and CXCL16 have been shown to bind to PS exposed at the RBC membrane (27). To analyze if these receptors influence the induction of PS exposure of RBCs, we performed flow chamber experiments. Supplemental Figure 6 demonstrates that neither blood from Cd36knockout mice nor treatment of Cd36-WT blood with inhibitory antibodies against CD36 and CXCL16 influences PS exposure of RBCs (Supplemental Figure 6, B-D).

Fas receptor-mediated cell-cell contact between platelets and $R B C s$ is essential for PS exposure of RBCs upon thrombus formation. Fas receptor (FasR, CD95) signaling in RBCs is known to induce an apoptotic signal via activation of caspase 3 leading to PS externalization in human RBCs (28). To investigate if this apoptotic mechanism plays a role in initiating PS exposure of RBCs, we initially confirmed FasR expression of RBCs (Supplemental Figure 6E). Unexpectedly, FasR expression on RBCs was increased in the presence of resting and activated platelets (Supplemental Figure 6F). The incubation of PRP with RBCs under static conditions showed significantly reduced annexin $\mathrm{V}$ binding to RBCs when we blocked the FasR of RBCs by antibody treatment upon platelet stimulation with ADP (100\% [PRP + RBCs] vs. $151.6 \%$ $\pm 16.1 \%[\mathrm{PRP}+\mathrm{RBCs}+\mathrm{ADP}]$ vs. $107.9 \% \pm 6.0 \%[\mathrm{PRP}+\mathrm{RBCs}$ + ADP + FasR antibody]; Figure 6A). To analyze the effects of FasR inhibition and reduced PS exposure of RBCs on platelet activation, we examined annexin $\mathrm{V}$ binding to platelets. In fact, PS exposure of ADP- and CRP-stimulated platelets was strongly reduced in the presence of RBCs treated with FasR-blocking antibody (Figure 6B). Moreover, annexin $\mathrm{V}$ binding to activated platelets was significantly enhanced in the presence of RBCs compared with activated platelets alone (Figure 6B), suggesting that RBCs amplify PS exposure of activated platelets. In the presence of RBCs, inhibition of FasR significantly reduced the endogenous thrombin potential (ETP) after stimulation of platelets with thrombin (Figure 6, C and D), while the FasR-inhibiting antibody had no effect in PRP alone (data not shown). To investigate the 
A
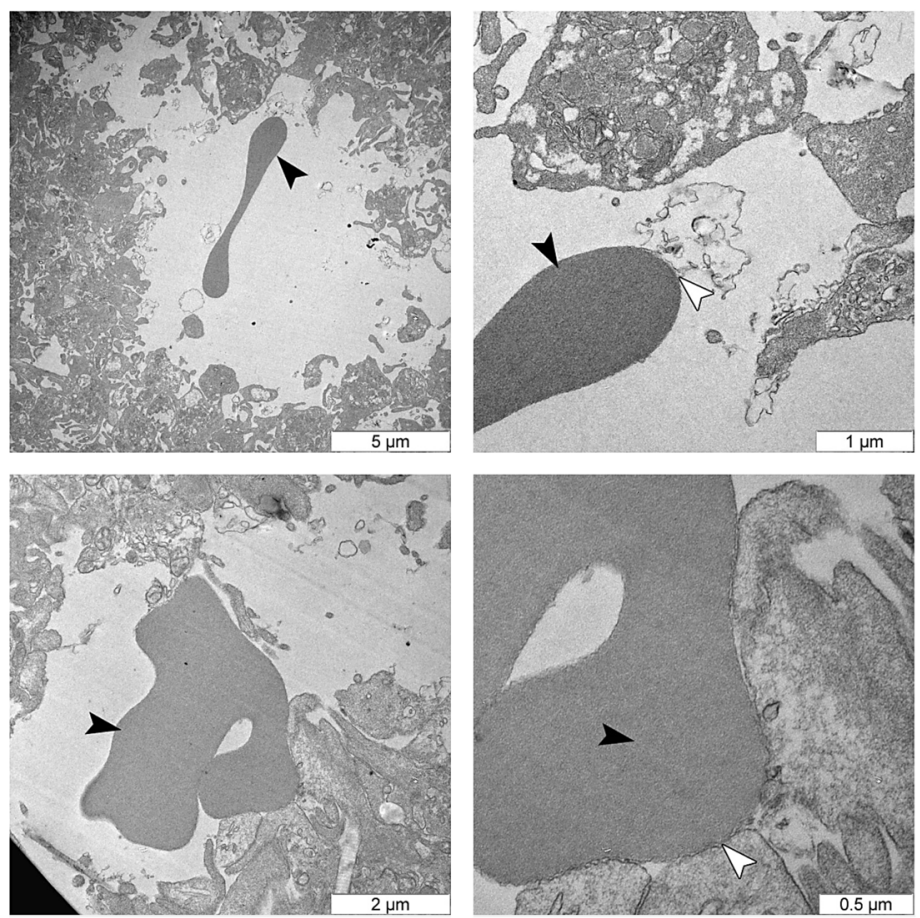

B
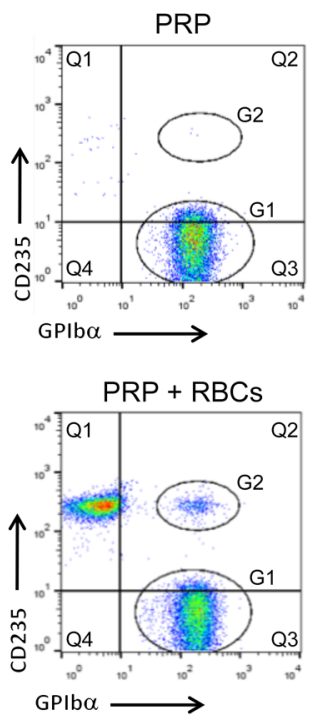

impact of FasR on RBCs under more physiological conditions, we performed flow chamber experiments and found significantly reduced thrombus formation under flow when we inhibited FasR only on RBCs (Figure 6, E and F). Accordingly, annexin V binding to RBCs and platelets was significantly reduced, as measured by flow cytometry (Figure 6, G and H). However, thrombus formation and PS exposure of RBCs was shown to be not dependent on caspases because experiments performed in the presence of a pan-caspase inhibitor did not alter surface coverage of thrombi, thrombus volume, or annexin V binding to RBCs (Supplemental Figure 6, $\mathrm{G}$ and $\mathrm{H}$ ).

To analyze if platelets have an activated phenotype in the presence of RBCs, we determined P-selectin exposure (degranulation marker) and activated integrin $\alpha \operatorname{IIb} \beta 3$ (PAC-1 binding) by flow cytometry (Supplemental Figure 7, A and B). We did not detect any
Figure 5. Cell-cell contact between platelets and RBCs is crucial for RBC-platelet interaction. (A) Transmission electron microscopy of thrombi. Overview (left images) and image details (on the right) are shown. RBCs (black arrowheads) and regions of cell-cell contact (white arrowhead) with platelets are highlighted. Scale bars as indicated $(n=3)$. (B) Representative dot blots from PRP incubated with RBCs using cell-specific antibodies. Gate 1 (G1) shows platelets. Gate 2 (G2) shows aggregates of RBCs and platelets. (C) Annexin V binding to RBCs as determined by flow cytometry $(n=7)$. $s / n$, supernatant. CRP (agonist) concentration $=5 \mu \mathrm{g} / \mathrm{ml}$. Data are the mean \pm SEM. ${ }^{* * *} P<0.001$ by Student's $t$ test.

platelet activation in the presence of RBCs. The same results were obtained in the presence of WBCs (Supplemental Figure 7, C-E).

To analyze whether FasR on RBCs has any impact on arterial thrombosis in vivo, we analyzed WT mice treated with FasR-blocking antibody $(20 \mu \mathrm{g} /$ mouse) in a thrombosis model where injury of mesenteric arterioles was induced by $\mathrm{FeCl}_{3}$. Platelet adhesion and thrombus formation at sites of vessel injury were induced by topical application of $20 \% \mathrm{FeCl}_{3}$ on exposed mesenteric arterioles. Complete vessel occlusion occurred in control mice within $1,420 \pm 142.6$ seconds, with an occlusion rate of $100 \%$. In contrast, we never observed occlusion of mesenteric arterioles in mice treated with the inhibitory FasR antibody, suggesting that these mice are protected against arterial thrombosis (Figure 6, I and J, and Supplemental Videos 5 and 6). In contrast, hemostasis, as analyzed by tail bleeding experiments, was not altered in mice that received FasR-blocking antibody (Figure 6K). Taken together, these results clearly show that interfering with platelet-RBC interactions by blocking FasR might be a promising new therapeutic strategy.

Platelet-membrane-bound Fas ligand serves as ligand for erythrocyte FasR to mediate plateletinduced PS exposure of RBCs. FasR is activated upon engagement by Fas ligand (FasL) (29). To investigate if FasL expressed on platelets is responsible for FasR activation on RBCs, we first analyzed FasL exposure on the platelet membrane (Figure 7A). While platelet activation did not lead to a significant increase in FasL externalization, we detected a significant increase in FasL expression on resting and activated platelets in the presence of RBCs. The presence of WBCs did not alter FasL expression of resting platelets but did alter that of activated platelets (Supplemental Figure 7E). In another set of experiments, platelet adhesion on immobilized FasR showed an increase of ADP-stimulated adherent platelets that was significantly reduced when platelets were treated with human decoy receptor 3 (hDcR3), while no differences were observed upon platelet adhesion to collagen (Figure 7, B-D). hDcR3 binds to FasL without any signal transduction capabilities and prevents 
A

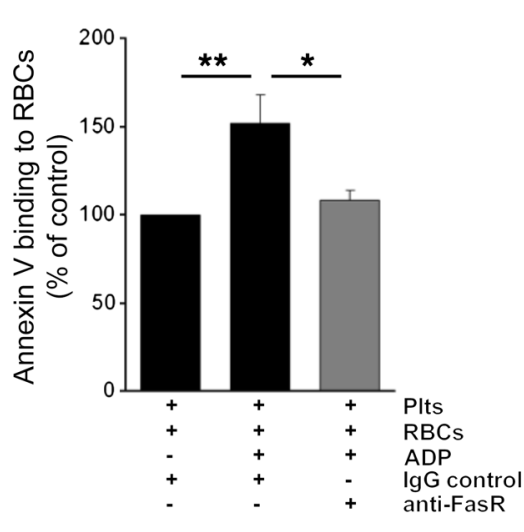

B

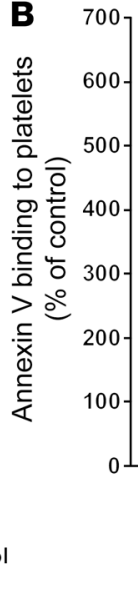

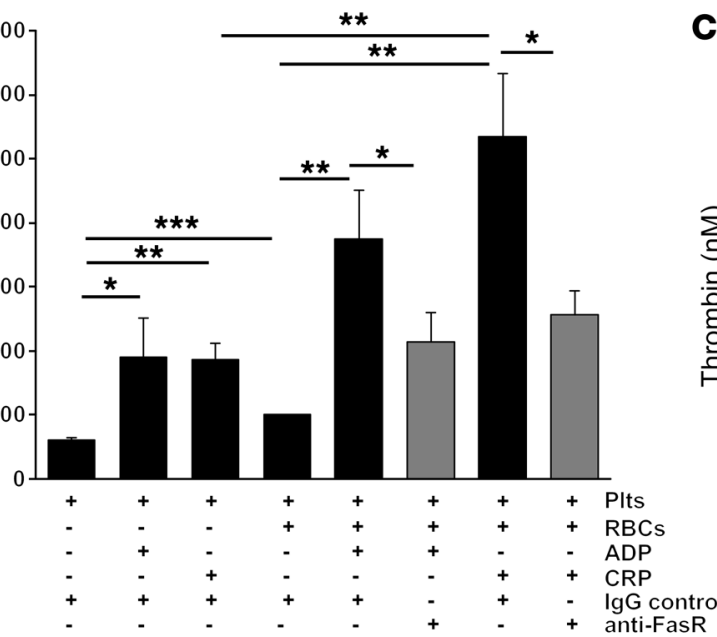

$\mathbf{E}$
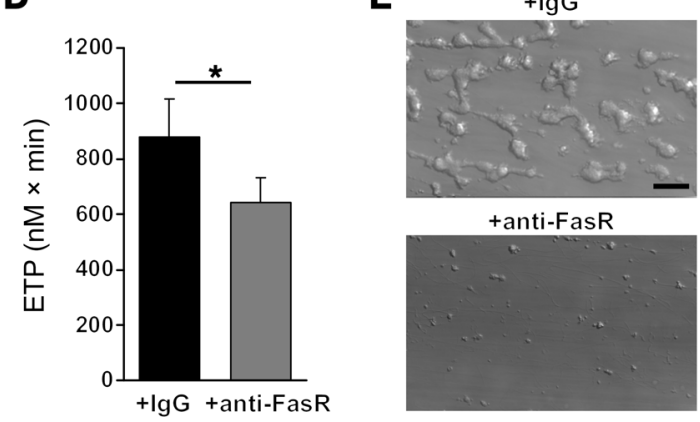

I

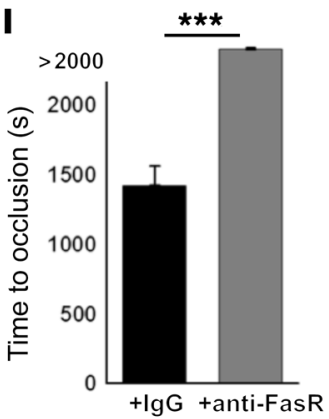

$\mathbf{F}$

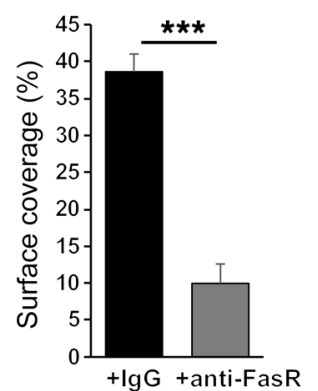

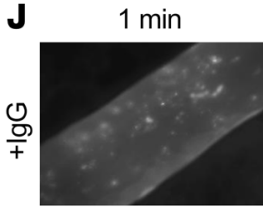

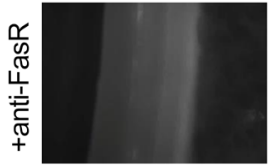

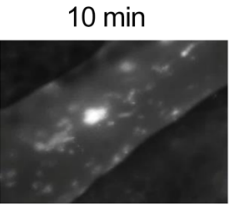

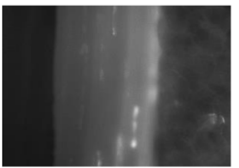

$20 \mathrm{~min}$

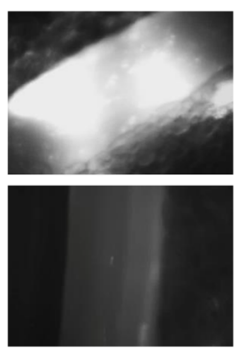

C

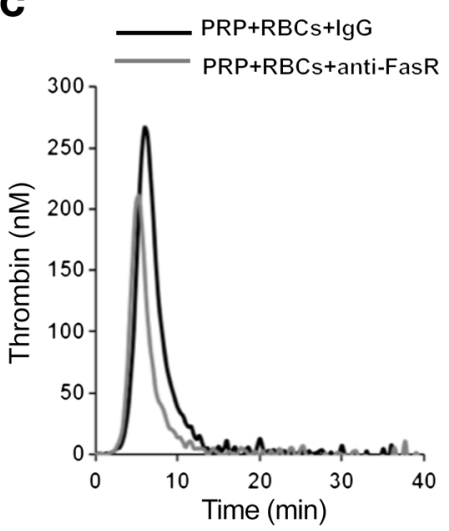

G

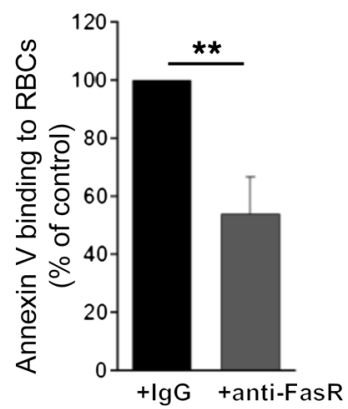

H

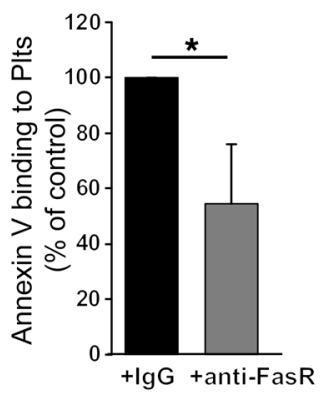

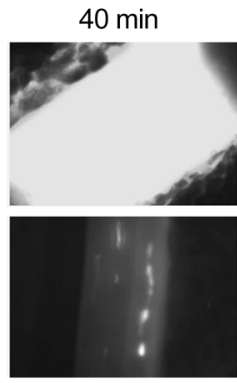

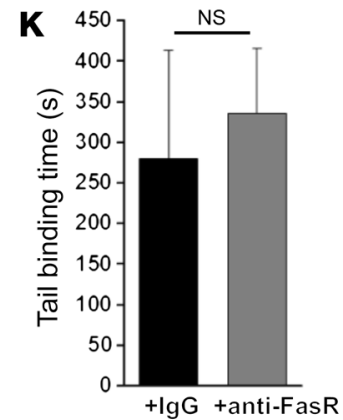

Figure 6. Cell-cell contact between platelets and RBCs via FasR (CD95). (A) Effects of FasR-blocking antibody on PS exposure of RBCs in the presence of ADP-stimulated platelets. $(n=9)$. (B) Platelet PS exposure was determined by flow cytometry $(n=4-6)$. (C and D) Thrombin-induced thrombin generation in PRP supplemented with RBCs in the presence and absence of FasR-blocking antibody was measured by calibrated automated thrombogram. (C) Representative thrombin curves and (D) endogenous thrombin potential (ETP) ( $\mathrm{nM} \times \mathrm{min}$ ) are shown. (E-H) Flow chamber experiments with FasR-blocking antibody. (E) Representative images of thrombus formation. Scale bar: $50 \mu \mathrm{m}$. (F) Mean surface coverage per visual field ( $n=5)$. ( $\mathbf{G}$ and

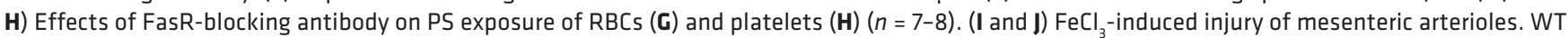
mice were injected with FasR-blocking antibody (anti-FasR, $20 \mu \mathrm{g} /$ mouse) and IgG ( $20 \mu \mathrm{g} / \mathrm{mouse}$ ) as control. Thrombus formation was monitored by intravital microscopy. (I) Time to irreversible occlusion (right panel). (J) Representative images at indicated time points ( $n=4-6 /$ group). (K) Tail bleeding times were determined in mice $(n=5-8 /$ group). FasR antibody was used at $10 \mu \mathrm{g} / \mathrm{ml}$. Agonist concentrations: ADP ( $10 \mu \mathrm{M})$ and CRP $(5 \mu \mathrm{g} / \mathrm{ml})$. Data are the mean $\pm \mathrm{SEM} .{ }^{*} P<0.05 ;{ }^{* *} P<0.01 ;{ }^{* *} P<0.001$ by 1 -way ANOVA with Tukey's multiple-comparisons test (A and $\left.\mathbf{B}\right)$ or Student's $t$ test (D-K). NS, not significant; Plts, platelets.

FasR-FasL interactions by competitively binding to membranebound FasL (30). Accordingly, annexin V binding to RBCs was significantly reduced when ADP-stimulated platelets were incubated with RBCs in the presence of hDcR3 (Figure 7E). Under flow conditions, platelet adhesion and 3-dimensional thrombus formation were significantly reduced in hDcR3-treated whole blood (Figure 7, F and G). In addition to a reduced surface cov- erage, we found significantly reduced thrombus volume when we perfused fluorescently labeled platelets through the chamber and measured integrated fluorescence intensity (IFI) (Figure $7 \mathrm{H})$. Consequently, the number of RBCs in thrombi was reduced and annexin $\mathrm{V}$ binding to RBCs showed a significant reduction of PS externalization in samples from thrombi derived from hDcR3-treated blood (Figure 7, I and J). 
A

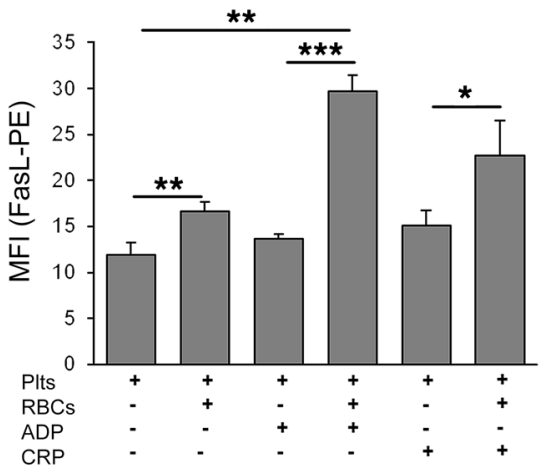

C

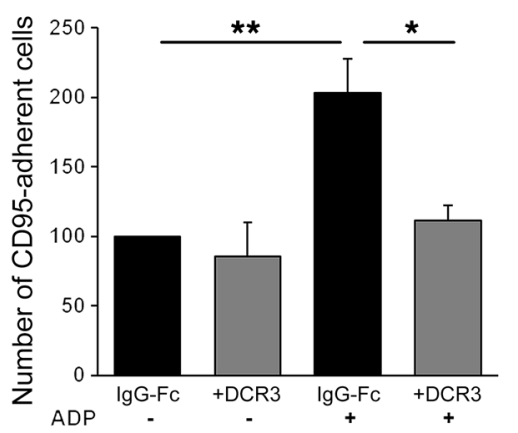

F $\operatorname{lgG}-\mathrm{Fc}$
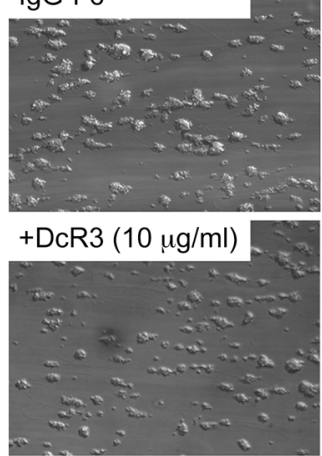

H

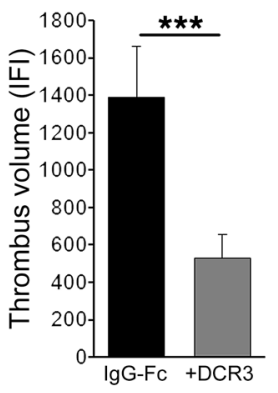

I
B

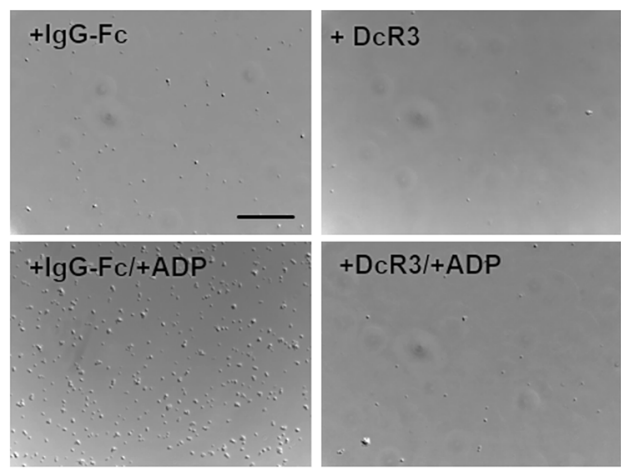

D
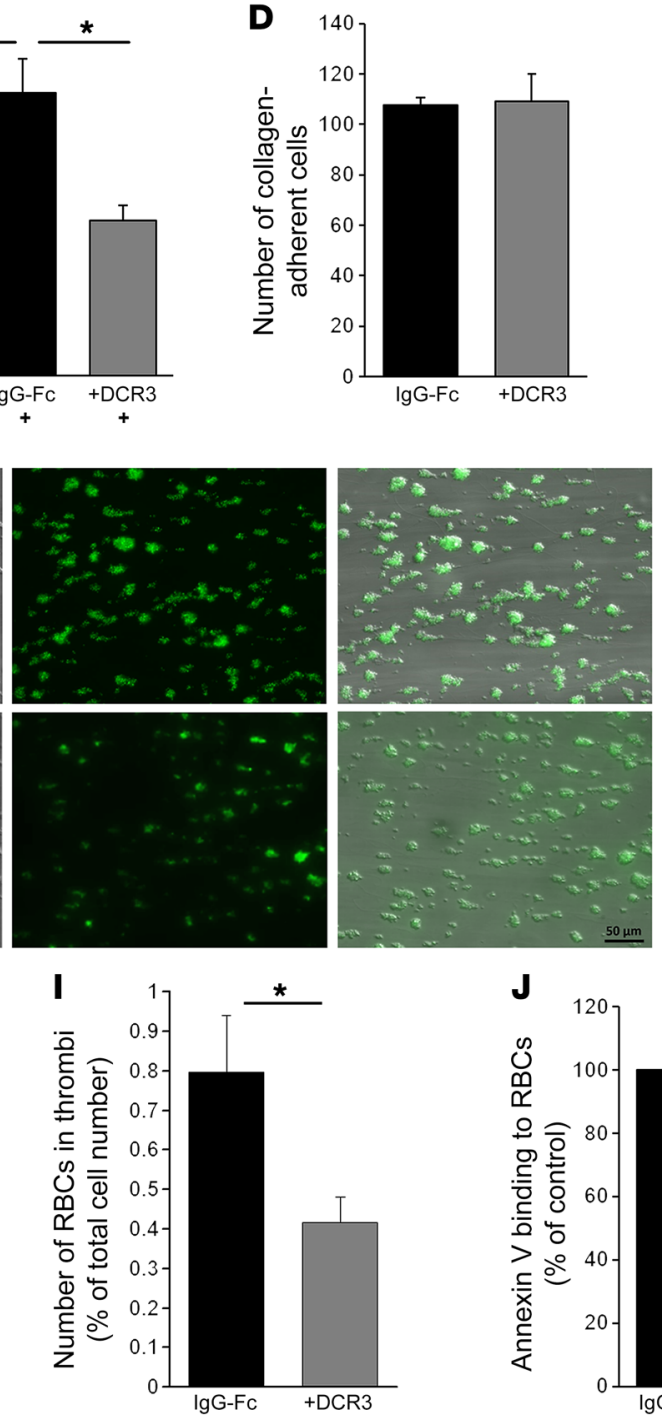

E
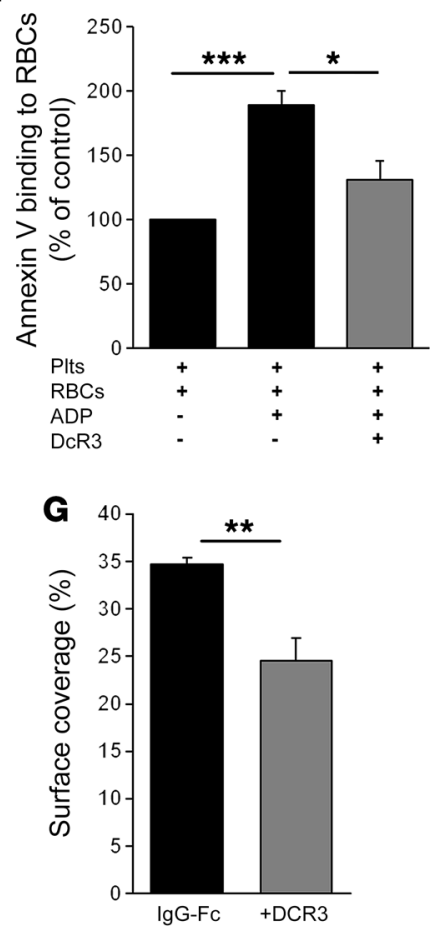

Figure 7. FasL serves as ligand for FasR to mediate platelet-induced activation of RBCs. (A) Exposure of FasL at the platelet membrane after incubation of platelets with ADP $(10 \mu \mathrm{M})$ and CRP $(5 \mu \mathrm{g} / \mathrm{ml})$ in the absence and presence of RBCs was determined by flow cytometry $(n=4-10)$. MFI, mean fluorescence intensity. (B and C) Platelets stimulated with ADP $(10 \mu \mathrm{M})$ were allowed to adhere to immobilized FasR $(50 \mu \mathrm{g} / \mathrm{ml})$. Representative images (B) and quantification (C) of adherent platelets in the presence of hDcR3 or control lgG-Fc $(10 \mu \mathrm{g} / \mathrm{ml})$. hDcR3, human decoy receptor 3. Scale bar: $50 \mu \mathrm{m}$. (D) No effect of hDcR3 on platelet adhesion on collagen $(200 \mu \mathrm{g} / \mathrm{ml})$ was observed. $(n=5)$. (E) Platelets were activated with ADP $(10 \mu \mathrm{M})$, pretreated with hDcR3 $(10 \mu \mathrm{g} / \mathrm{ml})$, and incubated with RBCs. Effect of hDcR3 treatment on PS exposure of RBCs was determined $(n=10)$. $(F-I)$ Aggregate formation of hDcR3-treated $(10 \mu \mathrm{g} / \mathrm{ml})$ platelets on collagen under flow using a shear rate of $1,000 \mathrm{~s}^{-1}$. Controls received IgG-Fc. (F) Representative phase-contrast (left panel) and fluorescence images with mepacrinelabeled platelets (middle panel, overlay right panel) at the end of the perfusion period. Scale bar: $50 \mu \mathrm{m}$. (G and $\mathbf{H})$ Mean surface coverage and relative platelet deposition after treatment with hDcR3, as measured by integrated fluorescence intensity (IFI) per visual field is shown $(n=6)$. (I) Cells of thrombi were isolated by Accutase treatment and number of RBCs was quantified by flow cytometry $(n=6)$. (J) PS exposure of RBCs treated with hDcR3 or control IgG-Fc was determined by flow cytometry $(n=6)$. Controls were treated with IgG-Fc protein $(n=8)$. Data are the mean \pm SEM. ${ }^{*} P<0.05 ;{ }^{* *} P<0.01 ;{ }^{* *} P<0.001$ by Student's $t$ test (D and G-J) or 1-way ANOVA with Tukey's multiple-comparisons test (A, C, and E). PIts, platelets. 
A
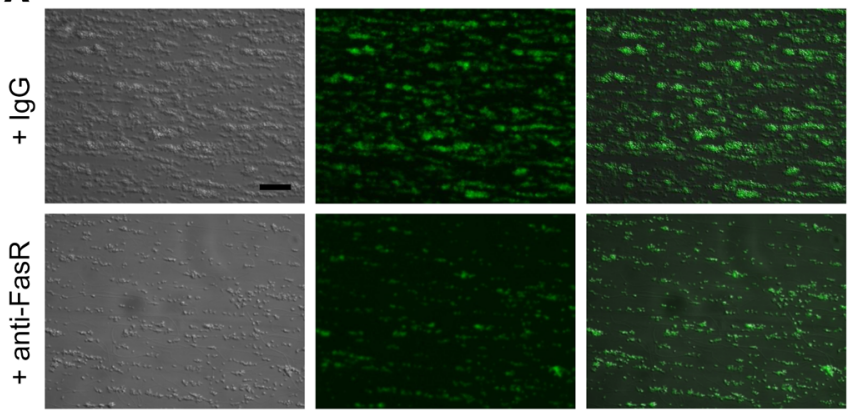

C
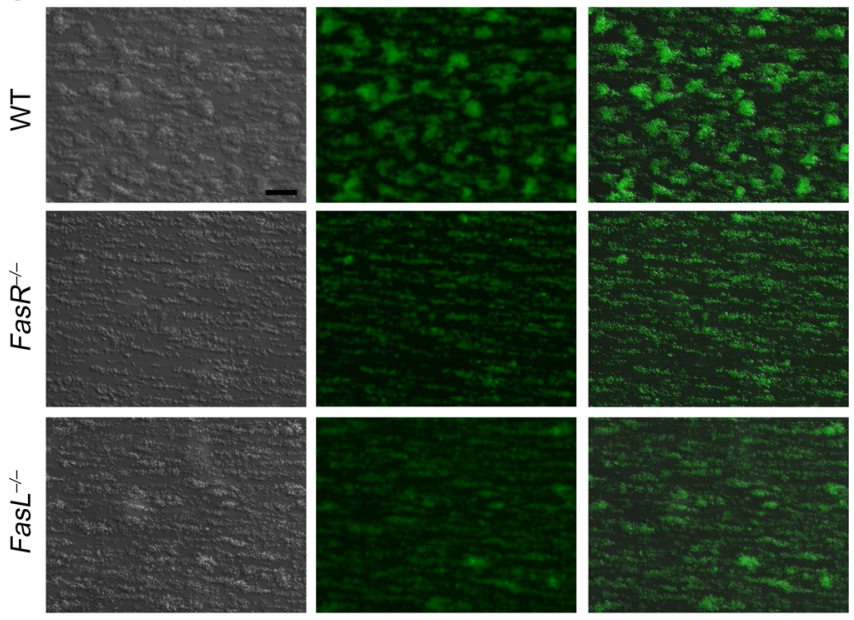

$\mathbf{F}$
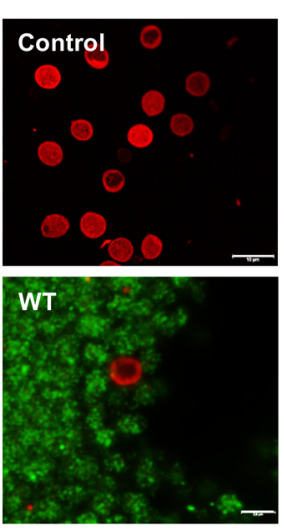

G
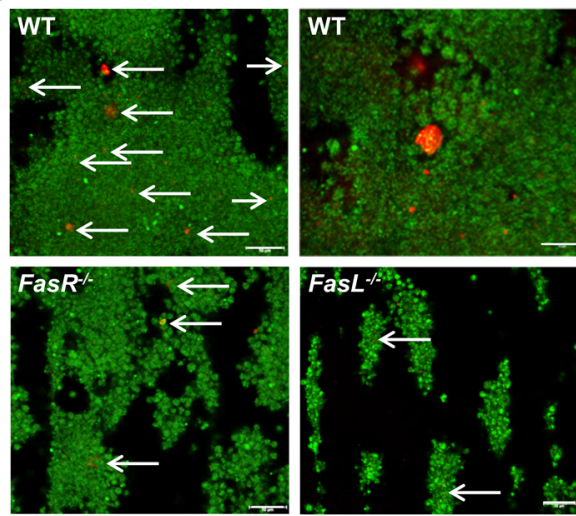

B

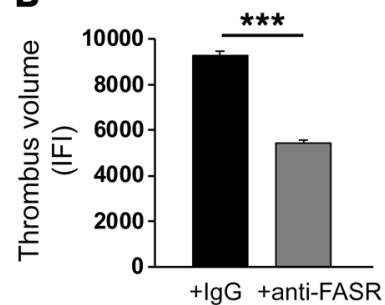

D

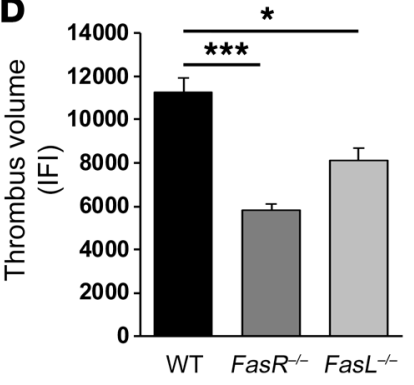

E

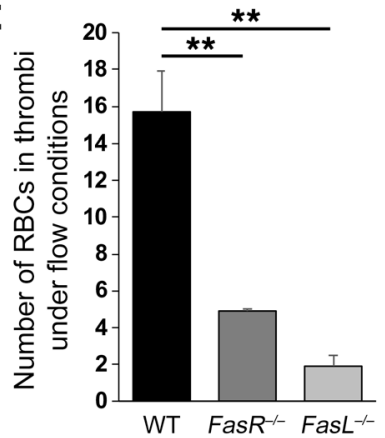

Figure 8. Genetic deletion of FasR and FasL reduces thrombus formation in vitro. (A and B) Flow chamber experiments with whole blood from WT mice treated with antibodies as indicated. (A) Representative images of thrombus formation. (B) Relative platelet deposition (IFI) per visual field ( $n=$ 4). (C and D) Aggregate formation under flow. Platelets from either WT or FasL ${ }^{-/-}$mice were incubated with WT RBCs, and WT platelets with $\mathrm{FasR}^{-/-} \mathrm{RBC}$ s were used. Representative images (C) and thrombus volume (D) are shown ( $n$ = 4-6). (E-G) Detection of RBCs and platelets in thrombi of WT, FasR ${ }^{-/-}$, or FasL $L^{-1-}$ mice $(n=3)$. (E) Number of RBCs in thrombi under flow conditions. (F) Staining of isolated RBCs (TER-119 antibody, red; upper panel) and of RBCs and platelets in thrombi (RBCs: TER-119 antibody, red; platelets: GP9 antibody, green; lower panel). (G) Representative images of RBCs in thrombi from WT, FasR ${ }^{-/-}$, or FasL ${ }^{-/-}$mice. Arrows indicate RBCs. Data are the mean \pm SEM. ${ }^{*} P<0.05 ;{ }^{* *} P<0.01$; ${ }^{* * *} P<0.001$ by Student's $t$ test (B) or 2-way ANOVA with Tukey's multiple-comparisons test ( $\mathbf{D}$ and E). Scale bars: $50 \mu \mathrm{m}$ (A and $\mathbf{C}$ ) and $10 \mu \mathrm{m}$ (F and $\mathbf{G})$.
Genetic deletion of FasR or FasL is responsible for altered PS exposure and thrombus formation in vitro and in vivo. To confirm the inhibitory experiments with FasR antibody and hDcR3, we analyzed FasL- and FasR-knockout mice. First, we measured platelet and RBC counts, hematocrit, and basal levels of PS exposure at the RBC membrane in knockout mice and found no significant alterations between the different mouse lines (Supplemental Figure 8, A-C).

Given that we analyzed human platelets with FasR antibody and hDcR3, we now analyzed thrombus formation under flow with murine blood and FasR-inhibiting antibody to confirm the human data (Figure 8A). Significantly decreased formation of 3-dimensional thrombi was found when we perfused whole blood supplemented with FasR antibody through the chamber using a shear rate of $1,700 \mathrm{~s}^{-1}$ (Figure 8, A and $\mathrm{B}$ ). In addition, thrombus formation and thrombus volume were significantly reduced when we perfused fluorescently labeled FasL-knockout $\left(\mathrm{FasL}^{-/-}\right)$platelets and WT RBCs or WT platelets and FasR-knockout (FasR $\left.{ }^{-/-}\right) \mathrm{RBCs}$ through the chamber and measured IFI (Figure 8, C and D). Moreover, we determined the number of RBCs in thrombi using the RBC marker TER-119 (red) and the platelet marker GP9 (green). As shown in Figure 8, E-G, the number of RBCs in thrombi was significantly reduced when we perfused whole blood from $\mathrm{FasL}^{-/-}$ or $\mathrm{FasR}^{-/-}$mice through the chamber.

Annexin $\mathrm{V}$ binding to RBCs and platelets in the absence or presence of RBCs was measured by flow cytometry under static conditions. As shown in Figure 9, the incubation of ADP-activated platelets from WT or FasL ${ }^{-/-}$mice with WT RBCs as well as WT 
A

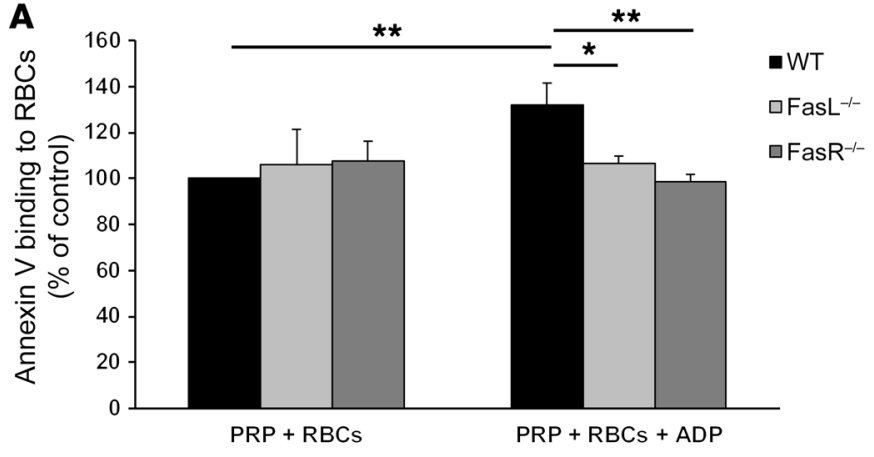

C

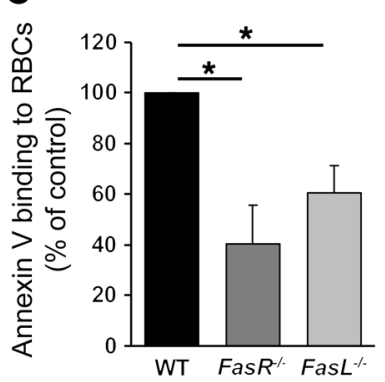

G

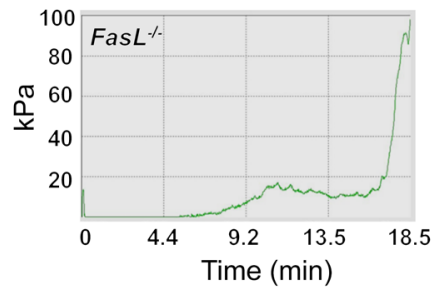

H

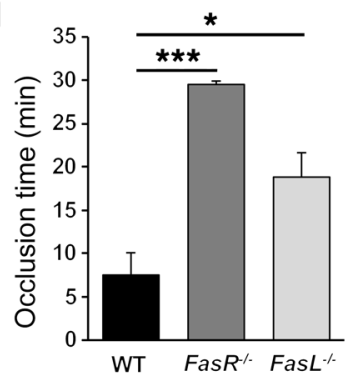

B

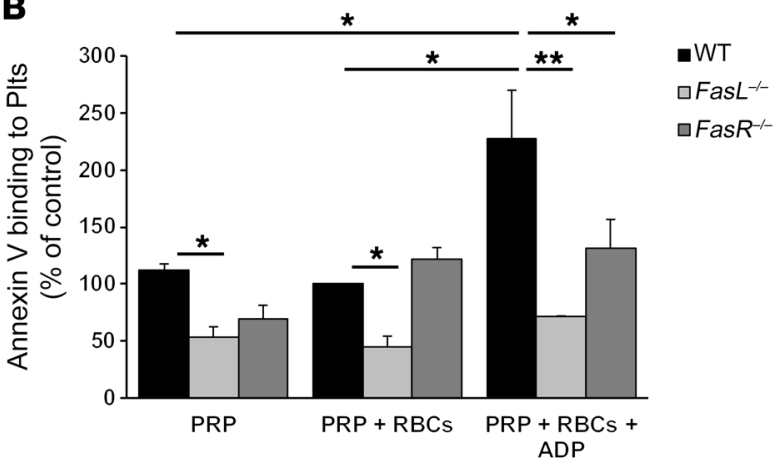

E
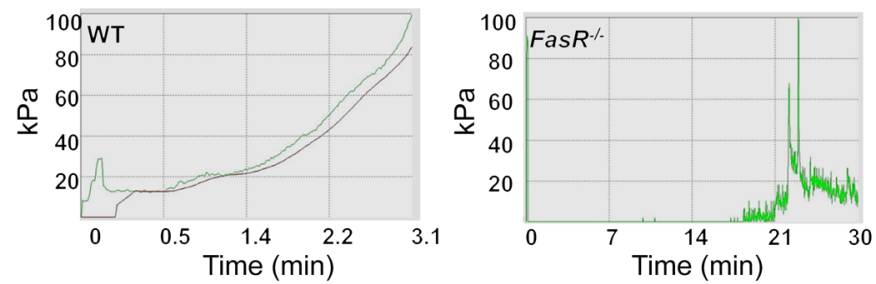

Figure 9. Genetic deletion of FasR and FasL reduces PS exposure in vitro. (A and B) Effects of FasL and FasR deficiency on PS exposure of (A) RBCs and (B) platelets in vitro. Platelets from either WT or FasL ${ }^{-/-}$mice were incubated with WT RBCs; WT platelets were incubated with Fas $R^{-1-}$ RBCs $(n=4)$. (C and $\left.\mathbf{D}\right)$ Flow cytometry of annexin V binding to RBCs $(\mathbf{C})$ and platelets $(\mathbf{D})$ isolated from thrombi $(n=5)$. (E-C) Thrombus formation and capillary occlusion in the flow chamber system T-TAS using PRP and RBCs. Time until full occlusion of the capillary was monitored. PRP from WT mice was incubated with either WT (E) or Fas $R^{-1-}$ RBCs (F) and compared to PRP from Fas $L^{-1-}$ mice and WT RBCs (C). (H) Occlusion times $(n=4-6)$. A shear rate of $1,700 \mathrm{~s}^{-1}$ was used. Data are the mean \pm SEM. ${ }^{*} P<0.05 ;{ }^{* *} P<0.01 ;{ }^{* *} P<0.001$ by 2 -way ANOVA with Tukey's multiple-comparisons test $(\mathbf{A}$ and $\mathbf{B})$ or 1 -way ANOVA with Dunnett's multiplecomparisons test $(\mathbf{C}-\mathbf{H})$. Plts, platelets.

platelets and FasR $^{-/}$RBCs led to a significant decrease in PS exposure of RBCs (Figure 9A) and platelets (Figure 9B). Moreover, resting $\mathrm{FasL}^{-/}$platelets displayed decreased PS exposure in PRP alone and in the presence of WT RBCs.

Accordingly, annexin $\mathrm{V}$ binding to RBCs (Figure 9C) and platelets (Figure 9D) isolated from thrombi in the flow chamber was significantly reduced when either Fas $R^{-/} \mathrm{RBCs}$ and WT platelets or $\mathrm{FasL}^{-1}$ platelets and WT RBCs were perfused through the chamber. Capillary occlusion in the Total Thrombus-formation Analysis System (T-TAS, Fujimori Kogyo Co., Ltd.; see supplemental methods) flow chamber confirmed significantly enhanced occlusion times when either $\mathrm{FasR}^{-/-} \mathrm{RBCs}$ and WT platelets or $\mathrm{FasL}^{-/}$platelets and WT RBCs were analyzed (Figure 9, E-H).

We next investigated the impact of FasR and FasL in sheardependent arterial thrombus formation in vivo. Topical application of $20 \% \mathrm{FeCl}_{3}$ on exposed mesenteric arterioles induced ves- sel injury that resulted in prompt initiation of thrombus formation (Figure 10A) and complete vessel occlusion in WT mice, while most of $\mathrm{FasR}^{-/-}$mice showed a delay in the initiation of thrombus formation and no occlusion at the end of the observation period of 40 minutes (Figure 10B). An even more pronounced phenotype was observed with $\mathrm{FasL}^{-1-}$ mice that showed no aggregate or thrombus formation at all; thus, no occlusion of the injured vessel was observed in these mice (occlusion time $>40$ minutes, Figure 10, A-D). Furthermore, thrombus size at different time points was significantly reduced in $\mathrm{FasR}^{-/-}$mice, while no thrombi were observed in $\mathrm{FasL}^{-1-}$ mice compared with WT controls (Figure 10E). To confirm the importance of platelet FasL in thrombus formation in vivo, we analyzed mice with a cell-specific knockout of FasL in platelets using PF4-Cre ${ }^{+} \mathrm{FasL}^{\mathrm{f} / \mathrm{l} l}$ mice. As shown in Figure 10F, initiation of thrombus formation in PF4-Cre ${ }^{+} \mathrm{FasL}^{\mathrm{A} / \mathrm{l}}$ mice was significantly delayed compared with PF4-Cre ${ }^{-}$FasL $^{\mathrm{t} / \mathrm{l}}$ mice that served 
as controls. Furthermore, 5 of $8 \mathrm{PF} 4-\mathrm{Cre}^{+} \mathrm{FasL}^{\mathrm{fl} / \mathrm{fl}}$ mice showed no occlusion of the injured vessel at the end of the observation period of 40 minutes, while $2 \mathrm{PF} 4-\mathrm{Cre}^{+} \mathrm{FasL}^{\mathrm{f} / \mathrm{fl}}$ mice showed a prolonged time to occlusion compared with PF4- $\mathrm{Cre}^{-} \mathrm{FasL}^{\mathrm{fl} / \mathrm{fl}}$ controls (Figure 10, $\mathrm{G}$ and $\mathrm{H}$ ), confirming the results obtained from $\mathrm{FasL}^{-/-}$mice.

To investigate the interaction between platelets and RBCs in another mouse model, we analyzed C57BL/6 mice after ligation of the inferior vena cava (IVC). C57BL/6 mice received murine platelets isolated from $\mathrm{C} 57 \mathrm{BL} / 6$ or $\mathrm{FasL}^{-/-}$mice and labeled with rhodamine $\mathrm{B}$, and RBCs isolated from $\mathrm{C} 57 \mathrm{BL} / 6$ or $\mathrm{FasR}^{-/-}$mice labeled with dichlorofluorescein (DCF). After ligation of the IVC, interactions between RBCs and platelets were defined as colocalization for at least 3 consecutive images (204 ms, Figure 10, I-K). Cell interactions were quantified per animal 30, 60, and 90 minutes after ligation (Figure 10J). C57BL/6 mice receiving FasR ${ }^{-/-} \mathrm{RBCs}$ or $\mathrm{FasL}^{-/-}$platelets showed significantly reduced platelet-RBC interactions 90 minutes after ligation compared with WT mice with RBCs and platelets from C57BL/6 (WT vs. FasR ${ }^{-/-}$RBCs: $10.75 \pm$ 1.548 vs. $4.25 \pm 0.25, P=0.003$; WT vs. FasL ${ }^{-1-}: 10.75 \pm 1.548$ vs. $5.25 \pm 1.25, P=0.0163$; Figure 10J). In a flow restriction model of the IVC, venous thrombus formation, as measured by thrombus weight, was not altered in mice treated with FasR-blocking antibody, although the incidence of thrombus formation was reduced: $30 \%$ incidence compared with $60 \%$ in IgG control mice (Figure $10 \mathrm{~L})$. Therefore, the incidence of thrombus formation (50\% of IgG-treated mice) rather than the thrombus weight represents differences between FasR antibody-and IgG-treated mice. In contrast, venous thrombus formation was significantly reduced in $\mathrm{FasL}^{-/-}$compared with control mice, while the incidence of thrombus formation was slightly enhanced in these mice (WT vs. FasL $L^{-1-}$ : $15.06 \mathrm{mg} \pm 3.91$ vs. $5.683 \pm 1.277, P=0.0446$; Figure $10 \mathrm{M})$.

Normal hemostasis upon FasR or FasL deficiency was observed when tail bleeding times of WT, $\mathrm{FasR}^{-/-}$, and $\mathrm{FasL}^{-/-}$mice were measured (Figure 10N). In line with these results, plateletspecific FasL-knockout mice were described to show no differences in bleeding times compared with controls as shown by Schleicher and colleagues (31).

FasL-FasR-mediated PS externalization of RBCs is relevant under pathological conditions. To investigate whether platelet-mediated PS externalization on the RBC membrane via FasL-FasR interaction influences arterial thrombosis in humans, we analyzed thrombi that were isolated from patients who underwent surgical thrombectomy (Table 1). Histological sections from these thrombi were stained with hematoxylin and eosin and characterized for thrombus morphology (Figure 11A). We found RBCs in small islands within the thrombus, as observed in mice after injury of the carotid artery (Figure 2D). TEM analysis of thrombi provided strong evidence for a direct contact between RBCs and platelets under pathological conditions (Figure 11B), as observed in thrombi that resulted from platelet adhesion and thrombus formation under flow (Figure 5A). Flow cytometric analysis of cell types within the thrombi revealed that $56.74 \% \pm 4.64 \%$ RBCs and $42.37 \% \pm 4.62 \%$ platelets were localized to the thrombi (Figure 11C). Next, we measured platelet activation and annexin $\mathrm{V}$ binding to platelets and RBCs isolated from these thrombi and compared the data with freshly isolated resting and activated platelets and RBCs from healthy volunteers. P-selectin exposure of platelets in the thrombi was significantly enhanced compared with resting platelets, but only moderately increased compared with CRP-activated platelets (Figure 11D). However, FasL externalization at the platelet membrane as well as PS exposure of RBCs isolated from patients' thrombi were strongly enhanced compared with CRPactivated platelets in the presence of fresh RBCs: $21.34 \pm 2.69$ PRP + fresh RBCs + CRP vs. $44.43 \pm 6.13$ platelets from patients (FasL), $6.95 \pm 1.79 \mathrm{PRP}+$ fresh RBCs + CRP vs. $23.36 \pm 2.91$ platelets from patient (annexin V), respectively (Figure 11, E and F). Interestingly, no differences were observed in annexin $\mathrm{V}$ binding to activated platelets (Figure 11G). Flow cytometric analysis of whole blood from patients revealed that P-selectin and PS exposure of platelets was significantly enhanced compared with healthy volunteers (Supplemental Figure 9), while no difference in FasL exposure was observed. We next compared RBC-rich thrombi and platelet-rich thrombi and found no alterations in FasL externalization (Figure $11 \mathrm{H}$ ). However, annexin $\mathrm{V}$ binding to RBCs in platelet-rich thrombi was significantly enhanced compared with PS exposure of RBCs in RBC-rich thrombi (Figure 11I). PS externalization of platelets was significantly higher in RBC-rich thrombi (Figure 11J). Taken together, these data provide the first evidence to our knowledge that cell-cell contact between platelets and RBCs via FasL-FasR interaction is also relevant under pathological conditions.

\section{Discussion}

In this study, we have shown that RBCs are essential for effective thrombus formation in vitro and in vivo, because a reduced number of RBCs results in attenuated hemostasis and prolonged occlusion times in anemic mice. Besides effects of RBCs on rheology conditions, a small population of RBCs is sufficient to support platelet-mediated thrombus formation by providing a procoagulant surface. Mechanistically, in a direct cell-cell contact between platelets and RBCs PS exposure of RBCs occurs, mediated through FasL-induced activation of FasR, followed by thrombin generation and platelet activation (Figure $11 \mathrm{~K}$ ). Inhibition or genetic deletion of FasR prevents 3-dimensional thrombus formation on collagen under flow ex vivo, arterial and venous thrombosis, and RBC-platelet interaction after IVC ligation in vivo, suggesting that FasL-FasR interaction is essential for thrombus formation. Mice with global FasL deficiency as well as platelet-specific loss of FasL confirmed the importance of platelet FasL for the induction of PS exposure at the RBC membrane and thrombus formation in vivo. High FasL and PS exposure of platelets and RBCs in arterial thrombi of patients provide evidence that FasL-FasR-mediated cell contact between platelets and RBCs represents a pathophysiological mechanism as well.

RBCs have been linked to thrombus formation for decades. They have been described to play a passive role in hemostasis by physical and chemical effects on the interaction between platelets and the vessel wall $(16,32)$. In flowing blood, RBCs displace platelets to the vessel wall, to induce contact of platelets with prothrombotic extracellular matrix proteins exposed upon vessel injury. In the $\mathrm{FeCl}_{3}$ mouse model of arterial thrombosis, $\mathrm{RBCs}$ were shown to participate in thrombosis by supporting platelet adhesion to the intact endothelium (33). A reduced hematocrit is accompanied by prolonged bleeding times in humans and experimental animals, providing a pathophysiologically reasoned rationale to reduce the 
A

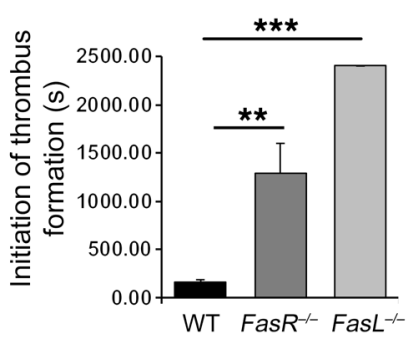

B

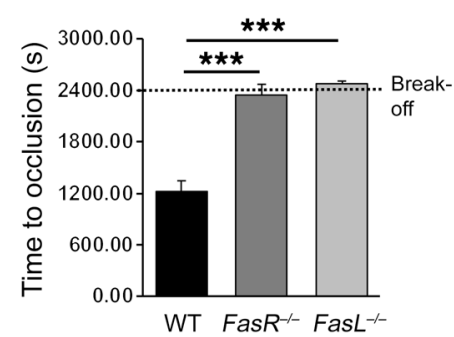

C

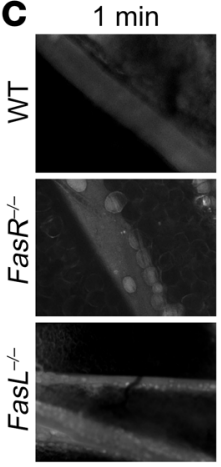

$10 \mathrm{~min}$
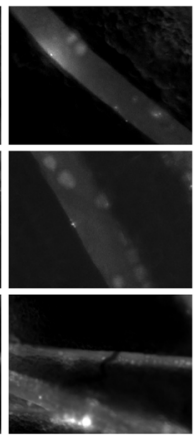

$20 \mathrm{~min}$
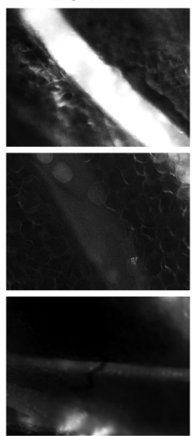

$40 \mathrm{~min}$

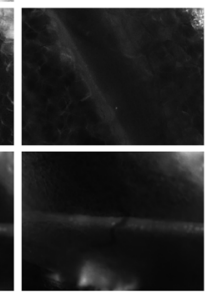

D

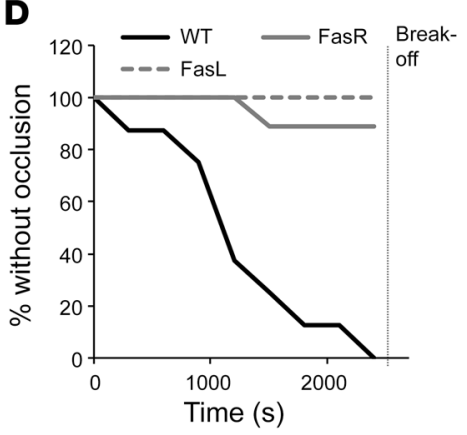

E

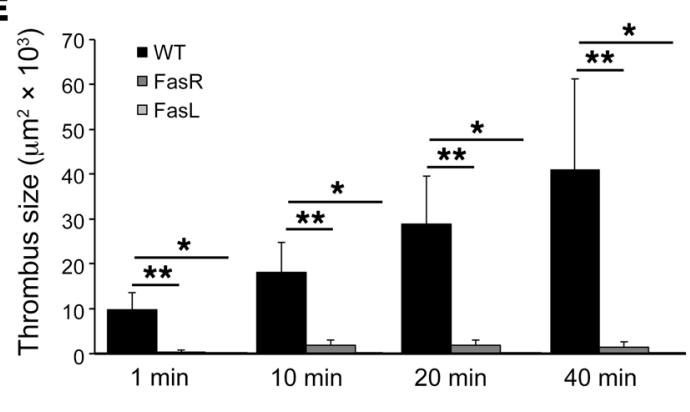

$\mathbf{F}$

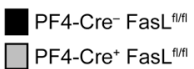

G
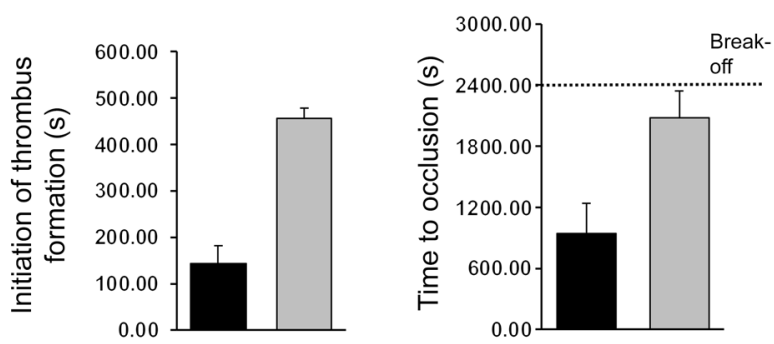

J

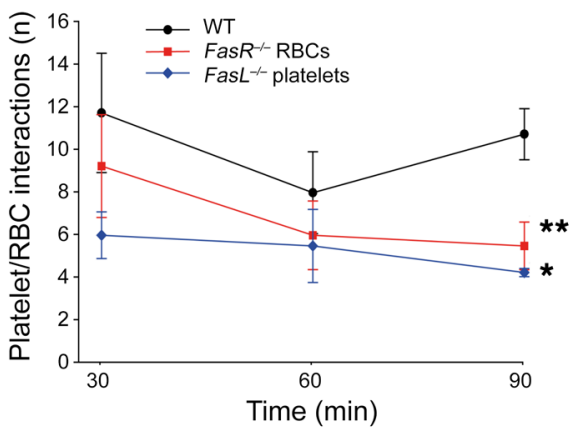

$\mathbf{L}$

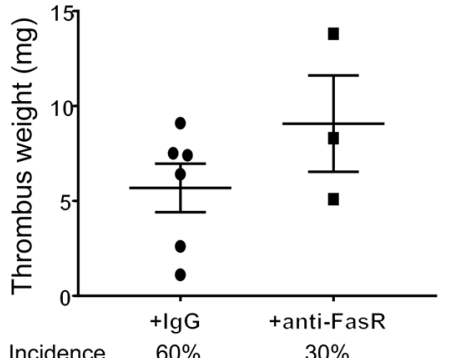

Incidence
M

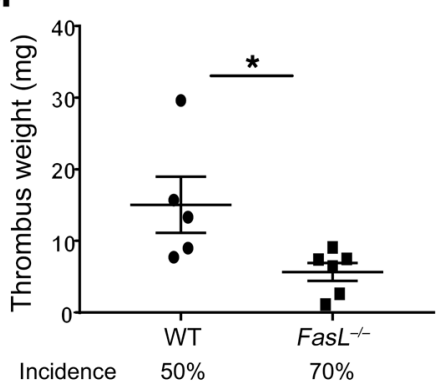

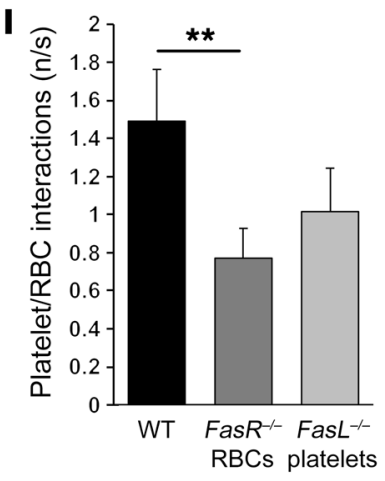

K

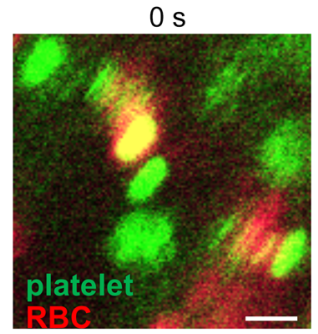

$8 \mathrm{~s}$
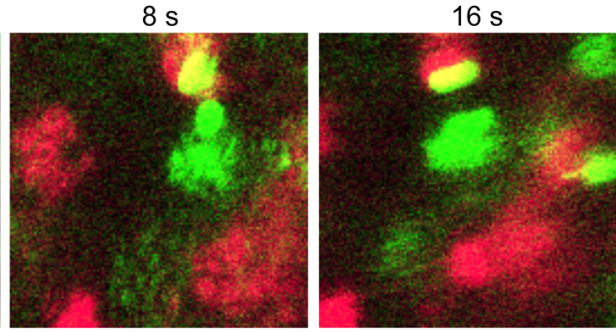

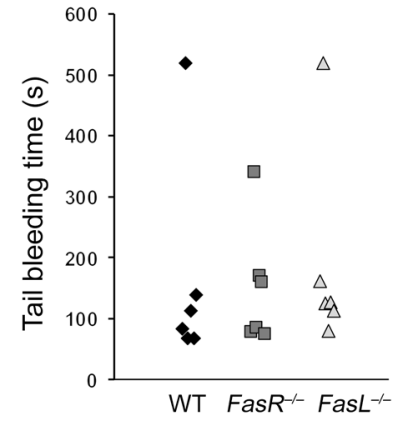


Figure 10. Genetic deletion of FasR and FasL reduces PS exposure and thrombus formation in vivo. (A-E) $\mathrm{FeCl}_{3}$-induced injury of mesenteric arterioles in WT, FasR $R^{-/-}$, or FasL ${ }^{-/-}$mice ( $n=5$-9/group). Beginning of thrombus formation (A), time to irreversible occlusion (B), representative intravital microscopy images (C), percentage of mice with no occlusion (D) (WT vs. Fas $R^{-1-}: P=0.0002$; WT vs. FasL ${ }^{-1-}: P=0.0011$ ), and thrombus size $(\mathbf{E})$ are shown. The break-off was set at $\mathbf{4 0}$ minutes after vessel injury when no occlusion occurred. (F-H) $\mathrm{FeCl}_{3}$-induced injury of mesenteric arte-

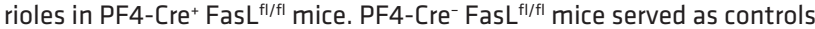
(F) Beginning of thrombus formation, (G) time to irreversible occlusion, and percentage of mice with no occlusion $(\mathbf{H}, P=0.003)$ are shown $(n=8 /$ group). (I-K) Platelet-RBC interactions after ligation of the inferior vena cava (IVC) were analyzed by intravital epifluorescence microscopy. Mice received murine platelets from $\mathrm{C} 57 \mathrm{BL} / 6$ or $\mathrm{FasL}^{-/-}$mice labeled with rhoda-

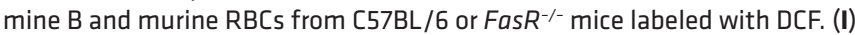
Number of platelet-RBC interactions per second (WT vs. Fas $R^{-/-}$RBCs: $P=$ 0.008 ; WT vs. Fas $L^{-/}$platelets: $P=0.072$ ). (J) Cell interaction was quantified (90 minutes: WT vs. Fas $R^{-/-}$RBCs: $P=0.003$; WT vs. Fas $L^{-/}$platelets: $P=0.0163)$. (K) Representative images of platelet-RBC interactions in WT mice 60 minutes after IVC ligation $(n=4)$. Scale bar: $10 \mu \mathrm{m}$. ( $L$ and M) Venous thrombus formation was investigated in a flow restriction model of the IVC and thrombus weight was determined: (L) IgG vs. FasR antibody-treated mice $(P=0.2814)$; $(\mathrm{M}) \mathrm{FasL}^{-1-}$ compared with WT mice $(P$ $=0.0446$ ). (N) Tail bleeding times were determined ( $n=6 /$ group). Data are the mean \pm SEM. ${ }^{*} P<0.05 ;{ }^{* *} P<0.01 ;{ }^{* *} P<0.001$ by Student's $t$ test $(\mathbf{F}, \mathbf{G}$, and $\mathbf{M})$, 1-way ANOVA with Dunnett's multiple-comparisons test (A, $\mathbf{B}, \mathbf{I}, \mathbf{J}$, and $\mathbf{N}$ ), log-rank (Mantel-Cox) test ( $\mathbf{D}$ and $\mathbf{H}$ ), or 2-way ANOVA with Sidak's multiple-comparisons test (E).

risk of bleeding by normalizing the hematocrit with $\mathrm{RBC}$ transfusions $(15,17,18,34)$. In this study, the analysis of thrombus formation with PRP supplemented with fixed RBCs (Figure 3D and Supplemental Figure 1) suggests that RBCs not only influence rheology but play an active role in supporting thrombus formation. Different mechanisms for RBCs and their role in hemostasis have been described. RBCs release ADP, ATP, and NO and thus might influence hemostasis by platelet activation (19-22). In 1961, Gaarder and colleagues found ADP released by RBCs to be relevant for platelet adhesion (20) and platelet aggregation (35). More recent data link RBCs with PS exposure (28) and suggest prothrombotic properties of RBCs $(23,24,36)$. Here, we directly provide evidence for the contribution of RBCs to thrombin generation because the supply of RBCs to PRP significantly increased the height of the thrombin peak and shortened the time when thrombin generation was initiated (Figure 4, H-J). However, less is known so far about the mechanisms of PS exposure of RBCs. Red cell ICAM- 4 was suggested to be a novel ligand for integrin $\alpha \operatorname{IIb} \beta 3$ on platelets $(37,38)$. This was strengthened by inhibitory experiments with decreased thrombin formation (39). Moreover, adhesion of RBCs to immobilized platelets depends on PS exposure of RBCs and the PS receptors CD36 and CXCL16 (27). To date, it is still not clear how RBCs come into first contact with platelets to induce FasL/FasR signaling. However, decreased incorporation of $\mathrm{RBCs}$ into a thrombus was observed when the interaction between ICAM- 4 and integrin $\alpha \operatorname{IIb} \beta 3$ was inhibited (39).

PS exposure of RBCs led to increased annexin $\mathrm{V}$ binding to platelets (Figure 6, B and H). The activation of platelets by RBCplatelet interactions has already been shown by different groups. In contrast to this study, the authors provided evidence for enhanced integrin activation, P-selectin exposure, thromboxane production, and ADP release when RBCs were present in platelet suspensions $(22,40,41)$. Here, we did not detect increased P-selectin exposure or integrin activation (Supplemental Figure 7). However, a direct mechanism for the induction of PS exposure on RBCs in a growing thrombus is currently unknown; nor has a direct contribution of RBCs to arterial thrombus formation by PS exposure, thrombin generation, or platelet activation been shown to date. Thus, we believe that the role of the FasL-FasR interaction in mediating platelet-RBC contacts upon adhesion to the injured vessel and arterial and venous thrombus formation is a completely novel element in platelet activation and aggregate formation. Here, we show that the death receptor FasR (CD95) on RBCs is essential for thrombus formation, while inhibition or genetic deletion of FasR protects mice from arterial thrombosis. FasL is a type II transmembrane protein that belongs to the tumor necrosis factor (TNF) family and binds to FasR to induce apoptosis $(29,42,43)$. Furthermore, FasL-FasR interactions play an important role in the regulation of the immune system and the progression of cancer (44-47). FasL is expressed by activated T cells and natural killer cells and induces apoptosis of target cells via FasR (48). Activated platelets expose FasL on their surface and induce apoptosis in Fas-positive tumor cells in vitro (49) and in a mouse model of stroke in vivo (31). This study reveals that platelet FasL is a ligand for FasR on the RBC membrane critical for the cell-cell interaction between RBCs and platelets. Interestingly, the expression of FasL on the platelet membrane (Figure 7A) as well as FasR expression on RBCs (Supplemental Figure 6) had already increased under resting conditions when platelets and RBCs were coincubated, suggesting that the cells influence the expression of receptors of the other cell. hDcR3 is a TNF-R1- and FasR-related death receptor and does not transduce apoptotic signals but competes with the death receptors for ligand binding to inhibit apoptosis $(30,50)$. It is noteworthy that hDcR3-mediated reduction of thrombus formation and PS exposure of RBCs within the thrombus is significantly reduced, but to a lesser extent than that with FasR inhibition. The same observation was made with FasL $^{-/}$platelets (Figure 8, C and D), suggesting that another ligand on the platelet membrane might be able to activate FasR on RBCs. Interestingly, PS exposure of RBCs was shown to be not dependent on caspases (Supplemental Figure 6, G and H), although caspase activation following FasL binding to FasR has been shown to reflect a general mechanism to induce apoptosis in target cells, especially in the immune system (47) and in cancer (46). However, in RBCs there might be another mechanism that translates FasR activation to PS exposure. In acute leukemia, an alternative pathway through TRADD and TRAF initiates a phosphorylation cascade leading to the activation of JNK and phosphorylation of p53 and c-Jun, which induces apoptosis through multiple mechanisms including Bdl-2 family proteins (51). An acid sphingomyelinase is also activated in response to FasR activation, leading to ceramide production and cell stress that also might account for PS exposure at the RBC membrane (52).

In recent years, it has become increasingly evident that different cellular processes are involved in hemostasis and thrombosis $(53,54)$. In this study, we observed that blocking FasR with an antibody (Figure 6K) or deficiency of either FasL or FasR does not lead to impaired hemostasis (Figure 10N) but protects against 
Table 1. Characteristics of patients who underwent surgical thrombectomy

\begin{tabular}{|c|c|c|c|c|c|c|}
\hline ID & Patient (year of birth) & Date of sampling & Place of removal & Weight (kg) & Body height (cm) & Smoker \\
\hline 1 & female (1941) & May 25, 2014 & forearm arteries & 90 & 163 & ex \\
\hline 2 & female (1939) & May 30, 2014 & femoro-distal bypass & 51 & 162 & ex \\
\hline 3 & male (1945) & June 16, 2014 & common femoral artery & 40 & 174 & yes \\
\hline 4 & male (1954) & July 9, 2014 & femoro-popliteal bypass & 120 & 181 & ex \\
\hline 5 & male (1952) & July 11, 2014 & femoro-popliteal bypass & 70 & 172 & ex \\
\hline 6 & female (1929) & July 18, 2014 & femoro-popliteal bypass & 74 & 158 & no \\
\hline 7 & male (1954) & July 21, 2014 & forearm arteries & 72 & 173 & yes \\
\hline 8 & male (1961) & July 23, 2014 & femoral and popliteal artery & 75 & 180 & yes \\
\hline 9 & female (1939) & August 13, 2014 & femoro-popliteal bypass & 67 & 168 & yes \\
\hline 10 & female (1935) & August 29, 2014 & A. rad., A. uln. sin. & 61 & 171 & no \\
\hline 11 & male (1946) & October 4, 2014 & femoro-popliteal bypass & 92 & 183 & yes \\
\hline 12 & male (1960) & October 11, 2014 & common femoral artery & 68 & 167 & yes \\
\hline 13 & female (1934) & October 22, 2014 & forearm arteries & 79 & 170 & no \\
\hline 14 & male (1936) & November 1, 2014 & poplitea dex. & 76 & 169 & no \\
\hline 15 & male (1946) & November 1, 2014 & femoro-popliteal bypass & 92 & 183 & yes \\
\hline 16 & male (1953) & January 4, 2015 & iliac arteries & 72 & 180 & no \\
\hline 17 & male (1951) & January 4, 2015 & iliac arteries & 73 & 176 & no \\
\hline 18 & female (1943) & January 21, 2015 & fem-fem crossover bypass & 66 & 176 & no \\
\hline 19 & female (1939) & January 28, 2015 & forearm arteries & 53 & 158 & no \\
\hline 20 & female (1959) & February 8, 2015 & femoro-popliteal bypass & 37 & 161 & no \\
\hline 21 & male (1952) & April 26, 2015 & femoro-popliteal bypass & 69 & 172 & yes \\
\hline 22 & female (1930) & June 6, 2015 & femoral artery & NA & NA & no \\
\hline 23 & female (1940) & June 8, 2015 & radial artery & 74 & 164 & no \\
\hline 24 & male (1948) & June 10, 2015 & femoro-popliteal bypass & 77 & 182 & yes \\
\hline 25 & male (1939) & June 19, 2015 & femoro-popliteal bypass & 76 & 178 & no \\
\hline 26 & male (1951) & July 14, 2015 & brachial artery & 110 & 180 & yes \\
\hline 27 & male (1944) & July 29, 2015 & femoral artery & 87 & 190 & yes \\
\hline 28 & female (1944) & September 29, 2015 & femoral artery & 90 & 176 & no \\
\hline 29 & female (1958) & October 7, 2015 & femoral artery & 50 & 163 & yes \\
\hline
\end{tabular}

A. rad, arteria radialis; A. uln. sin., arteria ulnaris sinistra; poplitea dex., poplitea dextra.

arterial thrombosis, while anemia leads to increased occlusion times and impaired hemostasis after vessel injury (Figure 3, E and F). This suggests that the rheology-dependent impact of RBCs in thrombus formation is different from the FasL-FasR interaction between platelets and RBCs.

The analysis of thrombi isolated from patients by thrombectomy suggests that FasL-FasR-mediated interaction between platelets and RBCs might be relevant under pathological conditions. Polycythemia patients with high RBC counts as well as patients with inherited RBC abnormalities suffer from thrombotic complications and exhibit cardiac and cerebral thrombosis with abnormal PS exposure on RBCs (55-60). Moreover, a correlation between infarct size and hematocrit was shown in patients with carotid occlusion and completed strokes (61). Our studies did not confirm a relationship between increased numbers of RBCs and thrombus formation because perfusion of PRP and $8 \times 10^{6} \mathrm{RBCs} / \mu \mathrm{l}$ did not result in accelerated thrombus formation under flow (Supplemental Figure 1, D and E). Moreover, mice with increased hematocrit did not show altered hemostasis or differences in arterial thrombus formation in vivo (Figure 3, $\mathrm{H}$ and I). However, we performed analysis under conditions of intermediate shear (flow chamber: 1,000 $\mathrm{s}^{-1}$; carotid artery: 295-645 s $\mathrm{s}^{-1}$. It is tempting to speculate that pathological high shear rates might affect RBC-platelet interaction, as has been shown by Turitto and Weiss (16). Under pathological conditions, increased procoagulant activity of RBCs was measured in patients with sickle-cell disease and with nephrotic syndrome $(56,62)$. Interestingly, RBC-rich thrombi contain more inflammatory cells in patients with ST-elevation myocardial infarction with impaired myocardial reperfusion (63). So far, the consequences of hypoxia or atherosclerotic vessels with decreased vessel lumen and high shear rates for platelet-RBC interactions are not known but might also be relevant, e.g., upon myocardial infarction or stroke.

However, high levels of PS externalization on platelets and RBCs in arterial thrombi of patients might also be due to the apoptotic character of these cells because some patients had already suffered from arterial thrombosis for several hours or days (for details see Table 1). 
A

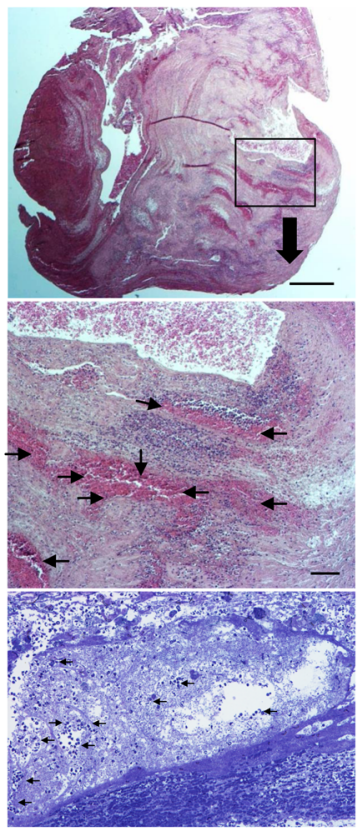

E

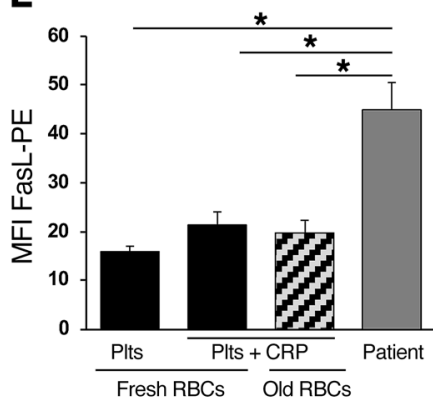

B

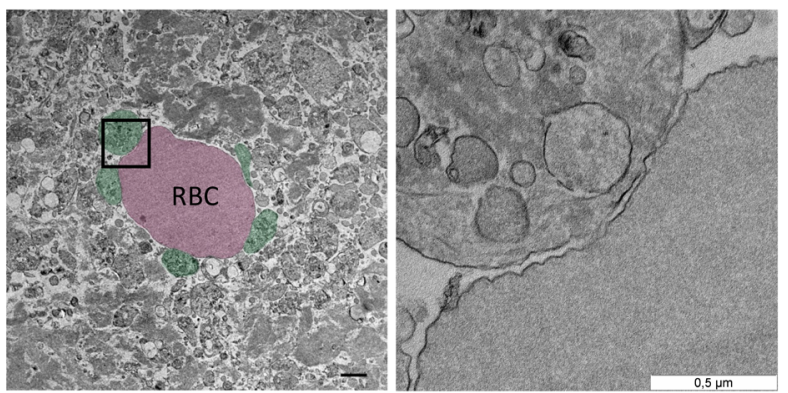

c

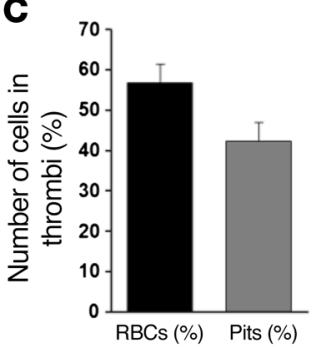

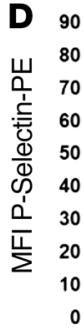

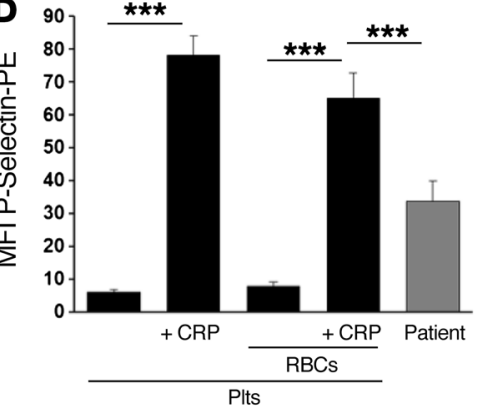

$\mathbf{F}$
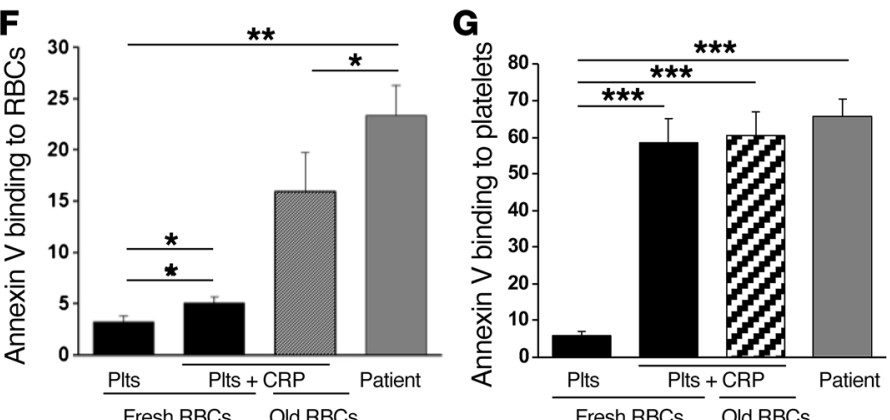

Fresh RBCs $\overline{\text { Old RBCs }}$
H

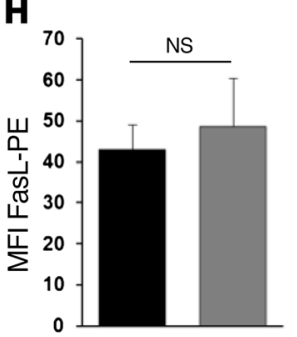

I

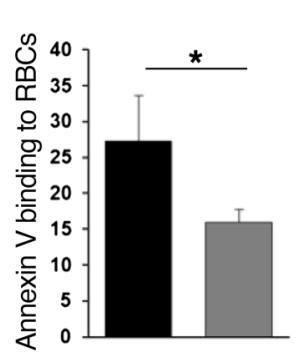

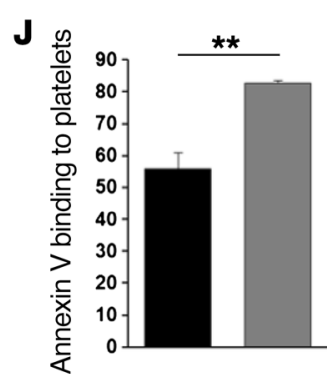

$\mathbf{K}$

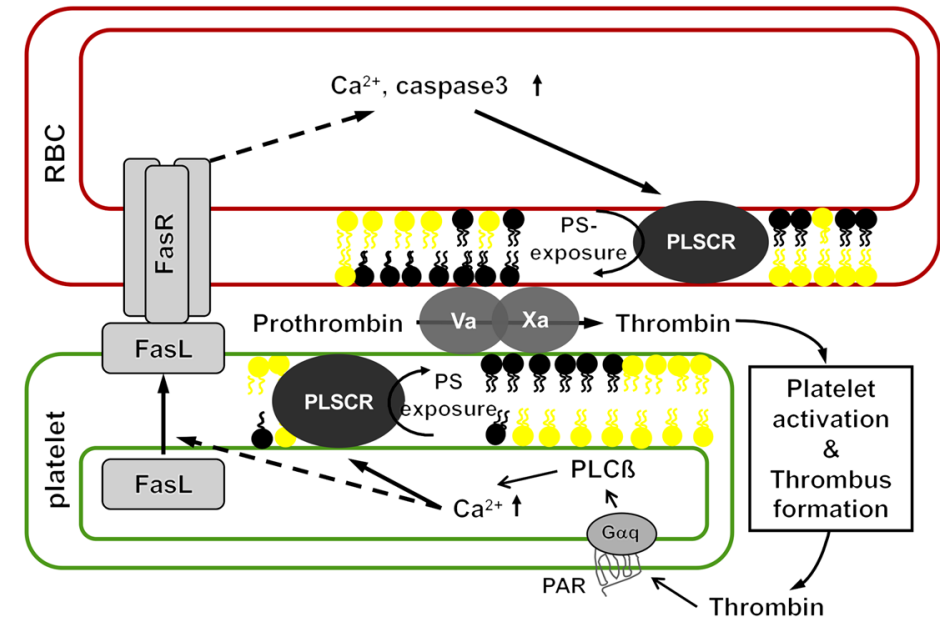

Plts $>$ RBC

RBC $>$ Plts
Figure 11. FasL/FasR-mediated PS exposure is relevant under pathological conditions. Human arterial thrombi from patients with thrombectomy were analyzed for cell content and cell markers.

(A) Representative images from hematoxylin and eosin staining of human thrombi. Overview (top, scale bar $500 \mu \mathrm{m}$ ) and details (middle panel, scale bar $100 \mu \mathrm{m}$ ) are shown. Black arrows mark RBCs. Representative images from toluidine blue staining of human thrombi (lower panel, ×200). (B) Representative transmission electron microscopic images of a human thrombus. Overview (left panel), with RBCs (red) and platelets (green), and details (right panel). Scale bars: $0.5 \mu \mathrm{m}$. (C) Quantification of RBCs and platelets and (D) determination of P-selectin exposure in human thrombi (patient) $(n=29)$. Platelets from healthy donors were activated with CRP and incubated with RBCs (controls, $n=9-11$ ). (E) Expression of FasL on platelets from human thrombi (patients) and healthy donors $(n=24)$. Determination of PS exposure of RBCs (F) and platelets (C) by flow cytometry. Isolated platelets and RBCs (fresh or older than 40 days after withdrawal) from healthy donors served as controls ( $n=$ 27). (H-J) Patients' samples were divided into platelet-rich (platelets $>$ RBCs) and RBC-rich (RBCs > platelets) thrombi and analyzed for FasL and PS exposure by flow cytometry. (H) Determination of FasL exposure in platelet-rich and RBC-rich human arterial thrombi ( $n=9-13)$. (I) PS exposure of RBCs was analyzed using annexin $\mathrm{V}(n$ $=11-14)$. (J) Annexin V binding to platelets in platelet-rich and RBCrich human thrombi $(n=4-6)$. (K) Tentative schematic illustration of RBCs and their impact on thrombus formation. CRP was used at $5 \mu \mathrm{g} / \mathrm{ml}$. Data are the mean \pm SEM. ${ }^{*} P<0.05 ;{ }^{* *} P<0.01 ;{ }^{* *} P$ $<0.001$ by Student's $t$ test ( $C$ and H-J) or 1-way ANOVA with Tukey's multiple-comparisons test (D-C) $\mathrm{MFI}$, mean fluorescence intensity; NS, not significant; PAR, proteaseactivated receptor; $\mathrm{PE}$, phycoerythrin; PLC $\beta$, phospholipase $C \beta$; PLSCR, phospholipid scramblase; PIts, platelets. 
Taken together, our results indicate that RBCs are essential for hemostasis and arterial as well as venous thrombosis. FasL-FasRmediated cell-cell contact between platelets and RBCs mediates PS externalization on the RBC membrane critical for platelet activation and adhesion upon thrombus formation. The potential mechanism underlying the RBC-platelet interaction identified here is summarized in Figure 11K. Thus, interfering with the FasL-FasR interaction might be an innovative and promising approach for a completely novel antithrombotic strategy.

\section{Methods}

Animals. Specific pathogen-free C57BL/6J mice were obtained from Janvier Labs. B6Smn.C3-Fas lld $/ \mathrm{J}$ mice were obtained from The Jackson Laboratory. Gene-targeted mice lacking CD36 and FasR (C57BL/6J.MRL-FAS ${ }^{\mathrm{lpr}}$ ), respectively, and the corresponding WT littermates were bred from breeder pairs and genotyped by PCR. FasL ${ }^{\mathrm{fl} / \mathrm{fl}}$ mice were provided by Ana Martin-Villalba (University of Heidelberg, Heidelberg, Germany) and crossed to PF4-Cre mice, which were purchased from The Jackson Laboratory (C57BL/6-Tg [Pf4-cre] Q3Rsko/J). PF4-Cre ${ }^{+}$FasL $^{\mathrm{fl} / \mathrm{fl}}$ mice or $\mathrm{PF}^{-} \mathrm{Cre}^{-} \mathrm{FasL}^{\mathrm{fl} / \mathrm{fl}}$ littermate controls were analyzed for thrombus formation. Experiments were performed with male and female mice aged 2-4 months.

Murine platelet preparation. Platelets were prepared as previously described $(64,65)$. Blood was collected into trisodium citrate and centrifuged at $250 \mathrm{~g}$ for 5 minutes at room temperature. To obtain PRP, the supernatant was centrifuged at $50 g$ for 6 minutes. PRP was washed twice at $650 \mathrm{~g}$ for 5 minutes and the pellet was resuspended in Tyrode's buffer (136 mM NaCl, $0.4 \mathrm{mM} \mathrm{Na}_{2} \mathrm{HPO}_{4}, 2.7 \mathrm{mM} \mathrm{KCl}, 12 \mathrm{mM}$ $\mathrm{NaHCO}_{3}, 0.1 \%$ glucose, $0.35 \%$ bovine serum albumin, $\mathrm{pH}$ 7.4) supplemented with prostacyclin $(0.5 \mu \mathrm{M})$ and apyrase $(0.02 \mathrm{U} / \mathrm{ml})$. Before use, platelets were resuspended in the same buffer and incubated at $37^{\circ} \mathrm{C}$ for 30 minutes.

Study approval. All animal experiments were conducted according to the Declaration of Helsinki and German law for the welfare of animals. The protocol was reviewed and approved by the local government authority (Heinrich-Heine-University Animal Care Committee and by the State Agency for Nature, Environment and Consumer Protection of North Rhine-Westphalia LANUV, Recklinghausen, NRW, Germany and by the local legislation on protection of animals Regierung von Oberbayern, Munich, Germany; Permit Numbers 84-02.05.20.12.A487, O 86/12, 84-02.05.40.16.073, and 84-02.04.2013.A210.

Experiments with human tissues and blood were reviewed and approved by the Ethics Committee of the Heinrich-HeineUniversity, who approved the collection and analysis of the tissue. Subjects provided informed consent prior to their participation in the study (patients' consent): Permit/Study Number 4669, ID No. 2014042327.

\section{Author contributions}

$\mathrm{HAH}, \mathrm{HS}$, JS, SM, MK, and ME designed the experiments, analyzed data, and wrote the manuscript. KS contributed to the design of experiments and data analysis. M. Spelleken carried out electron microscopic studies. WL performed RBC deformability experiments. KJ conducted thrombin measurements. LP performed IVC ligation experiments. CK, IK, SZ, KJK, M. Schaller, NSG, and AO performed all other experimental work.

\section{Acknowledgments}

We thank Bodo Steckel and Kathrin Schwierczek for excellent technical assistance, and Philipp Lang for providing FasR-knockout mice. This study was supported by grant Sonderforschungsbereich 1116 (TP A05) from the Deutsche Forschungsgemeinschaft, Düsseldorf, and the Forschungskommission of the Medical Faculty Düsseldorf (grant 2016/09 to M. Elvers).

Address correspondence to: Margitta Elvers, Department of Vascular and Endovascular Surgery, University Hospital Düsseldorf, Moorenstraße.5, 40225 Düsseldorf, Germany. Phone: 49.0.211.81.08851; Email: margitta.elvers@med.uni-duesseldorf.de.
1. Gawaz M, Langer H, May AE. Platelets in inflammation and atherogenesis. J Clin Invest. 2005;115(12):3378-3384.

2. Ruggeri ZM, Mendolicchio GL. Adhesion mechanisms in platelet function. Circ Res. 2007;100(12):1673-1685.

3. Murray CJ, Lopez AD. Mortality by cause for eight regions of the world: Global Burden of Disease Study. Lancet. 1997;349(9061):1269-1276.

4. Ruggeri ZM. Platelets in atherothrombosis. Nat Med. 2002;8(11):1227-1234.

5. Jackson SP. Arterial thrombosis--insidious, unpredictable and deadly. Nat Med. 2011;17(11):1423-1436.

6. Savage B, Saldivar E, Ruggeri ZM. Initiation of platelet adhesion by arrest onto fibrinogen or translocation on von Willebrand factor. Cell. 1996;84(2):289-297.

7. Nieswandt B, Varga-Szabo D, Elvers M. Integrins in platelet activation. J Thromb Haemost. 2009;7 Suppl 1:206-209.

8. Nieswandt B, Watson SP. Platelet-collagen interaction: is GPVI the central receptor? Blood. 2003;102(2):449-461.
9. Michelson AD. Antiplatelet therapies for the treatment of cardiovascular disease. Nat Rev Drug Discov. 2010;9(2):154-169.

10. Reed GL. Platelet secretory mechanisms. Semin Thromb Hemost. 2004;30(4):441-450.

11. Kay JG, Grinstein S. Phosphatidylserinemediated cellular signaling. Adv Exp Med Biol. 2013;991:177-193.

12. de Witt SM, Verdoold R, Cosemans JM, Heemskerk JW. Insights into platelet-based control of coagulation. Thromb Res. 2014;133 Suppl 2:S139-S148.

13. Hellem AJ, Borchgrevink CF, Ames SB. The role of red cells in haemostasis: the relation between haematocrit, bleeding time and platelet adhesiveness. Br J Haematol. 1961;7:42-50.

14. Ho CH. Increase of red blood cells can shorten the bleeding time in patients with iron deficiency anemia. Blood.1998;91(3):1094.

15. Livio M, Gotti E, Marchesi D, Mecca G, Remuzzi G, de Gaetano G. Uraemic bleeding: role of anaemia and beneficial effect of red cell transfusions. Lancet. 1982;2(8306):1013-1015.

16. Turitto VT, Weiss HJ. Red blood cells: their dual role in thrombus formation. Science.
1980;207(4430):541-543.

17. Blajchman MA, Bordin JO, Bardossy L, Heddle NM. The contribution of the haematocrit to thrombocytopenic bleeding in experimental animals. Br J Haematol. 1994;86(2):347-350.

18. Saniabadi AR, Lowe GD, Barbenel JC, Forbes CD. Further studies on the role of red blood cells in spontaneous platelet aggregation. Thromb Res. 1985;38(3):225-232.

19. Bergfeld GR, Forrester T. Release of ATP from human erythrocytes in response to a brief period of hypoxia and hypercapnia. Cardiovasc Res. 1992;26(1):40-47.

20. Gaarder A, Jonsen J, Laland S, Hellem A, Owren PA. Adenosine diphosphate in red cells as a factor in the adhesiveness of human blood platelets. Nature. 1961;192:531-532.

21. Mellion BT, Ignarro LJ, Ohlstein EH, Pontecorvo EG, Hyman AL, Kadowitz PJ. Evidence for the inhibitory role of guanosine $3^{\prime}, 5^{\prime}$-monophosphate in ADP-induced human platelet aggregation in the presence of nitric oxide and related vasodilators. Blood. 1981;57(5):946-955.

22. Valles J, et al. Erythrocytes metabolically enhance 
collagen-induced platelet responsiveness via increased thromboxane production, adenosine diphosphate release, and recruitment. Blood. 1991;78(1):154-162.

23. Noh JY, et al. Procoagulant and prothrombotic activation of human erythrocytes by phosphatidic acid. Am J Physiol Heart Circ Physiol. 2010;299(2):H347-H355.

24. Peyrou V, Lormeau JC, Hérault JP, Gaich C, Pfliegger AM, Herbert JM. Contribution of erythrocytes to thrombin generation in whole blood. Thromb Haemost. 1999;81(3):400-406.

25. Whelihan MF, Mann KG. The role of the red cell membrane in thrombin generation. Thromb Res. 2013;131(5):377-382.

26. Heemskerk JW, Vuist WM, Feijge MA, Reutelingsperger CP, Lindhout T. Collagen but not fibrinogen surfaces induce bleb formation, exposure of phosphatidylserine, and procoagulant activity of adherent platelets: evidence for regulation by protein tyrosine kinase-dependent $\mathrm{Ca}^{2+}$ responses. Blood. 1997;90(7):2615-2625.

27. Walker B, et al. Dynamic adhesion of eryptotic erythrocytes to immobilized platelets via platelet phosphatidylserine receptors. Am J Physiol, Cell Physiol. 2014;306(3):C291-C297.

28. Mandal D, Mazumder A, Das P, Kundu M, Basu J. Fas-, caspase 8-, and caspase 3-dependent signaling regulates the activity of the aminophospholipid translocase and phosphatidylserine externalization in human erythrocytes. J Biol Chem. 2005;280(47):39460-39467.

29. Schneider P, et al. Characterization of Fas (Apo1, CD95)-Fas ligand interaction. J Biol Chem. 1997;272(30):18827-18833.

30. Sheikh MS, Fornace AJ. Death and decoy receptors and p53-mediated apoptosis. Leukemia. 2000;14(8):1509-1513.

31. Schleicher RI, et al. Platelets induce apoptosis via membrane-bound FasL. Blood. 2015;126(12):1483-1493.

32. Ho CH. The hemostatic effect of packed red cell transfusion in patients with anemia. Transfusion. 1998;38(11-12):1011-1014.

33. Barr JD, Chauhan AK, Schaeffer GV, Hansen JK, Motto DG. Red blood cells mediate the onset of thrombosis in the ferric chloride murine model. Blood. 2013;121(18):3733-3741.

34. Eugster M, Reinhart WH. The influence of the haematocrit on primary haemostasis in vitro. Thromb Haemost. 2005;94(6):1213-1218.

35. Aursnes I, Gjesdal K, Abildgaard U. Platelet aggregation induced by ADP from unsheared erythrocytes at physiological $\mathrm{Ca}^{++}$-concentration. Br J Haematol. 1981;47(1):149-152.

36. Whelihan MF, Zachary V, Orfeo T, Mann KG.
Prothrombin activation in blood coagulation: the erythrocyte contribution to thrombin generation. Blood. 2012;120(18):3837-3845.

37. Hermand P, Gane P, Callebaut I, Kieffer N, Cartron JP, Bailly P. Integrin receptor specificity for human red cell ICAM-4 ligand. Critical residues for alphaIIbeta3 binding. Eur J Biochem. 2004;271(18):3729-3740.

38. Hermand $P$, et al. Red cell ICAM- 4 is a novel ligand for platelet-activated alpha IIbbeta 3 integrin. J Biol Chem. 2003;278(7):4892-4898.

39. Du VX, Huskens D, Maas C, Al Dieri R, de Groot PG, de Laat B. New insights into the role of erythrocytes in thrombus formation. Semin Thromb Hemost. 2014;40(1):72-80.

40. Santos MT, Vallés J, Aznar J, Pérez-Requejo JL. Role of red blood cells in the early stages of platelet activation by collagen. Thromb Haemost . 1986;56(3):376-381.

41. Vallés J, et al. Platelet-erythrocyte interactions enhance alpha(IIb)beta(3) integrin receptor activation and P-selectin expression during platelet recruitment: down-regulation by aspirin ex vivo. Blood. 2002;99(11):3978-3984.

42. Starling GC, Bajorath J, Emswiler J, Ledbetter JA, Aruffo A, Kiener PA. Identification of amino acid residues important for ligand binding to Fas. JExp Med.1997;185(8):1487-1492.

43. Suda T, Nagata S. Purification and characterization of the Fas-ligand that induces apoptosis. JExp Med.1994;179(3):873-879.

44. Li-Weber M, Krammer PH. Function and regulation of the CD95 (APO-1/Fas) ligand in the immune system. Semin Immunol. 2003;15(3):145-157.

45. O'Brien DI, Nally K, Kelly RG, O'Connor TM, Shanahan F, O'Connell J. Targeting the Fas/Fas ligand pathway in cancer. Expert Opin Ther Targets. 2005;9(5):1031-1044.

46. Peter ME, et al. The role of CD 95 and CD95 ligand in cancer. Cell Death Differ. 2015;22(4):549-559.

47. Strasser A, Jost PJ, Nagata S. The many roles of FAS receptor signaling in the immune system. Immunity. 2009;30(2):180-192.

48. Nagata S. Apoptosis by death factor. Cell. 1997;88(3):355-365.

49. Ahmad R, Menezes J, Knafo L, Ahmad A. Activated human platelets express Fas-L and induce apoptosis in Fas-positive tumor cells. J Leukoc Biol. 2001;69(1):123-128.

50. Pitti RM, et al. Genomic amplification of a decoy receptor for Fas ligand in lung and colon cancer. Nature. 1998;396(6712):699-703.

51. Schimmer AD, Hedley DW, Penn LZ, Minden MD. Receptor- and mitochondrial-mediated apoptosis in acute leukemia: a translational view.
Blood. 2001;98(13):3541-3553.

52. Cifone MG, et al. Apoptotic signaling through CD95 (Fas/Apo-1) activates an acidic sphingomyelinase. J Exp Med.1994;180(4):1547-1552.

53. Elvers M, et al. Impaired alpha(IIb)beta(3) integrin activation and shear-dependent thrombus formation in mice lacking phospholipase D1. Sci Signal. 2010;3(103):ra1.

54. Gowert NS, et al. Loss of Reelin protects mice against arterial thrombosis by impairing integrin activation and thrombus formation under high shear conditions. Cell Signal. 2017;40:210-221.

55. Chen S, et al. Enhanced aggregability of red blood cells of beta-thalassemia major patients. Am J Physiol. 1996;270(6 Pt 2):H1951-H1956.

56. Helley D, Eldor A, Girot R, Ducrocq R, Guillin MC, Bezeaud A. Increased procoagulant activity of red blood cells from patients with homozygous sickle cell disease and beta-thalassemia. Thromb Haemost. 1996;76(3):322-327.

57. Randi ML, Stocco F, Rossi C, Tison T, Girolami A. Thrombosis and hemorrhage in thrombocytosis: evaluation of a large cohort of patients (357 cases). J Med.1991;22(4-5):213-223.

58. Rossi C, Randi ML, Zerbinati P, Rinaldi V, Girola$\mathrm{mi}$ A. Acute coronary disease in essential thrombocythemia and polycythemia vera. J Intern Med. 1998;244(1):49-53

59. Ruf A, et al. In-vivo platelet activation correlates with red cell anionic phospholipid exposure in patients with beta-thalassaemia major. Br JHaematol. 1997;98(1):51-56.

60. Schafer AI. Bleeding disorders. Finding the cause. Hosp Pract (OffEd). 1984;19(11):88K, 88L, $88 \mathrm{M}$ passim.

61. Harrison MJ, Pollock S, Kendall BE, Marshall J Effect of haematocrit on carotid stenosis and cerebral infarction. Lancet. 1981;2(8238):114-115.

62. Gao C, et al. Procoagulant activity of erythrocytes and platelets through phosphatidylserine exposure and microparticles release in patients with nephrotic syndrome. Thromb Haemost. 2012;107(4):681-689.

63. Yunoki K, et al. Erythrocyte-rich thrombus aspirated from patients with ST-elevation myocardial infarction: association with oxidative stress and its impact on myocardial reperfusion. Eur Heart J. 2012;33(12):1480-1490.

64. Elvers $\mathrm{M}$, et al. Intracellular cyclophilin A is an important $\mathrm{Ca}(2+)$ regulator in platelets and critically involved in arterial thrombus formation. Blood. 2012;120(6):1317-1326.

65. Fotinos A, et al. Loss of oligophrenin1 leads to uncontrolled Rho activation and increased thrombus formation in mice. J Thromb Haemost. 2015;13(4):619-630. 